\title{
PHASE TRANSITIONS IN THE ONE-DIMENSIONAL COULOMB GAS ENSEMBLES
}

\author{
BY TATYANA S. TUROVA ${ }^{1}$ \\ University of Lund
}

\begin{abstract}
We consider the system of particles on a finite interval with pairwise nearest neighbours interaction and external force. This model was introduced by Malyshev [Probl. Inf. Transm. 51 (2015) 31-36] to study the flow of charged particles on a rigorous mathematical level. It is a simplified version of a 3-dimensional classical Coulomb gas model. We study Gibbs distribution at finite positive temperature extending recent results on the zero temperature case (ground states). We derive the asymptotics for the mean and for the variances of the distances between the neighbouring charges. We prove that depending on the strength of the external force there are several phase transitions in the local structure of the configuration of the particles in the limit when the number of particles goes to infinity. We identify 5 different phases for any positive temperature.

The proofs rely on a conditional central limit theorem for nonidentical random variables, which has an interest on its own.
\end{abstract}

1. Introduction. Coulomb gas distribution appears in a variety of mathematical models. Here, we focus on the one which describes the charges with nearest neighbour Coulomb interaction on an interval in a presence of external force. This model was introduced and studied recently by Malyshev [16], and then by Malyshev and Zamyatin [17], motivated by the fact that many electric phenomena are still not well understood, and "even might seem mysterious" ([17]).

Study of statistical mechanics of Coulomb gas has a long history. Coulomb gas models in dimension 2 are of a particular interest. Their connection to the Ginzburg-Landau theory of superconductivity and to random matrix theory is well explained in [19]. It appears that the Gibbs distribution of the Coulomb gas model is strongly related to the distribution of the eigenvalues of random matrices (see, e.g., [6] on a brief introduction into the typical methods in this area). The existence and universality of scaling limits for the eigenvalues of a random normal matrix are studied, for example, in [2,3] and [4] (see the reference therein).

The large deviations principle helps to study the configurations of the Coulomb gas particularly at low temperature, when the configurations which minimize the energy make the major contribution to the partition function. For the $d$ dimensional models of Coulomb gas the large deviations principle (at speed $N^{2}$ )

Received December 2016; revised July 2017.

${ }^{1}$ On leave from IMPB Russian Academy of Science Branch of KIAM RAS

MSC2010 subject classifications. 82B21, 82B26, 60F05.

Key words and phrases. Coulomb gas, phase transitions, Gibbs ensemble. 
is proved in [19] for a rather general situation. This result is used to determine the macroscopic distribution of the particles. Some linear statistics of the configurations are discussed in [6] (see, in particular, Example 1 in [6] for the dimension-one model.)

Recently, in [5] a precise asymptotic expansion of the free energy was derived, which allowed to establish the central limit theorem for the fluctuations of the linear statistics at any positive temperature.

The large deviations principle (at speed $N$ ) is established even for the twodimensional two-component plasma [13]. The latter model considers particles of both positive and negative charges with logarithmic interactions.

The one-dimensional models play an important role in statistical physics being the first rigorously studied cases. In a series of papers, Lenard ([8, 14, 15]) developed a method of Wiener integrals to study the partition function for the Gibbs distribution related to the one-dimensional Coulomb energy, which is given by

$$
H(q, \sigma)=-\sum_{i \neq j} \sigma_{i} \sigma_{j} \phi\left(\left|q_{i}-q_{j}\right|\right),
$$

where $q_{i} \in R$ is location of the $i$ th particle, and $\sigma_{i} \in\{-1,+1\}$ is its charge. The case $\phi(x)=x$ corresponds the (truly) one-dimensional Coulomb potential; it was studied in [8, 14, 15]. Already in [14], an effect of a constant external electric field was briefly discussed. The results of [8] and [15] on the thermodynamic limits and phase transitions were further developed; see, for example, [1], where the model of jellium [taking $\sigma_{i} \equiv-1$ in (1.1)] was treated as well. Results of [1] also confirmed a periodic structure of the limiting states in the presence of constant external field.

Another continuous one-dimensional gas model with Lennard-Jones-type potential [the pairwise interaction is $\phi(r)=\frac{1}{r^{\gamma}}-\frac{c}{r^{2}}, C>0$ ] is considered in [11] and [12]. It is proved that depending on the parameters of the model at the low temperature there are basically three different phases in the space occupied by the gas, firmly distinguished by the corresponding density of particles. The proofs in [11] and [12] rely on sophisticated combinatorial arguments concerning the entropy of the system.

The model of [16] which we consider here, is aimed to study the flow of charged particles in a network-like media, which might be approximated by a graph, that is, it is locally 1-dimensional. Therefore, we combine the features of dimension one, where the particles are assumed to be hardly aligned on an interval, and of "real" dimension 3, taking interactions in a form $\phi(x)=\frac{1}{x}$ [see (1.1)]; all particles have the same charges in our model. Already in [16], it was proved that at the zero temperature case (ground states) there are phase transitions in the structure of the configuration of charges under different strength of external force. Then a local structure of Gibbs configurations at positive temperature but without external force was analyzed in [17].

Here, we study how the local properties of configurations are changed in the presence of an external electric field. Simplicity of our model allows a "precise 
resolution": we are able to distinguish 5 different phases. We prove that for the weak force the charges remain to be densely and almost equally spaced over the entire interval, at the critical value of external force they occupy only a finite part of the interval, and when the force is above the critical value, all of the charges collapse in one end of the interval.

Note that here the phase transitions (along the strength of the external forth) are observed at any positive temperature, unlike, for example, in the model of [12], where similar transitions in the empirical density of particle are proved but only at low temperature. Therefore, model [16] provides a rare example of an exactly solvable model which, while being not realistic in a present form, still exhibits the properties of physical systems. Furthermore, the proved results were not yet reported even qualitatively in more complicated Coulomb gas models.

The major simplification of the present model is that only the nearest neighbours interactions are taken into account. This certainly is a crucial assumption for the presented exact solution. However, having understood this case one can get strong intuition about more complex models; we shall discuss some generalizations after we state the results below.

The methods we use here develop the probabilistic approach of [17] (and [7]) but now in an inhomogeneous setting. Notice also that the basic idea in the proof is still in a way close to the large deviations technique.

\section{Model and results.}

2.1. Model. Consider a system of $N+1$ identical particles on the interval $[0, L]$, whose locations are represented by a vector $\bar{y}=\left(y_{0}, \ldots, y_{N}\right)$ with ordered components:

$$
0=y_{0}<\cdots<y_{N}=L .
$$

The length of the interval plays no role, so we fix it from now on to be one:

$$
L=1 \text {. }
$$

Fixed values $y_{0}=0$ and $y_{N}=1$ mean here that at both ends there are particles with fixed positions.

For any vector (configuration)

$$
\bar{y} \in \mathcal{S}:=\left\{\left(y_{0}, \ldots, y_{N}\right): 0=y_{0}<\cdots<y_{N}=1\right\}
$$

define a function of potential

$$
U(\bar{y})=\beta \sum_{k=1}^{N} V\left(y_{k}-y_{k-1}\right)+\sum_{k=1}^{N} \int_{0}^{y_{k}} F_{s} d s,
$$

where the positive function $V$ represents a pairwise interaction between the particles, $\beta>0$ is a parameter, and function $F_{S}$ represents an external force at point $s \in[0,1]$. (Notice that here all the charges have the same sign.) 
ASSUMPTION 2.1. A pairwise Coulomb repulsive interaction is given by

$$
V(x)=\frac{1}{x}, \quad x>0 .
$$

The external force $F_{y}=F$ does not depend on the location $y$, but it is a function of the number of particles: $F=F(N)$.

Under Assumption 2.1, we can rewrite the potential (2.1) of a configuration $\bar{y}$ as

$$
U_{\beta, F}(\bar{y})=\beta \sum_{k=1}^{N} \frac{1}{y_{k}-y_{k-1}}+\sum_{k=1}^{N} F y_{k} .
$$

For a positive temperature $t>0$, the potential function $U_{\beta, F}(\bar{y})$ defines the following Gibbs density function:

$$
f(\bar{y} ; t)=\frac{1}{Z_{\frac{1}{t} U_{\beta, F}}(N)} e^{-\frac{1}{t} U_{\beta, F}(\bar{y})}, \quad \bar{y} \in \mathcal{S},
$$

where the normalizing factor (called the partition function) is

$$
Z_{\frac{1}{t} U}(N)=\int \cdots \int_{0<y_{1}<\cdots<y_{N-1}<1} e^{-\frac{1}{t} U(\bar{y})} d y_{1} \cdots y_{N-1} .
$$

For $N>2$, let $\bar{Y}=\left(Y_{0}=0, Y_{1}, \ldots, Y_{N-1}, Y_{N}=1\right)$ be a random vector on $\mathcal{S}$ with the Gibbs density (2.4). The entries of this vector represent positions of the particles on the interval: $N-1$ random and two fixed positions at both ends.

We shall study the asymptotic distribution of the inter-spaces between the particles, which are the random variables $Y_{k}-Y_{k-1}, 1 \leq k \leq N$, when $N \rightarrow \infty$.

When $F=0$, this model was treated in [17] [it is a particular case of part (a) of the following below theorem].

When $t \rightarrow 0$, the Gibbs distribution (2.4) converges to the one concentrated at the minimum of $U_{\beta, F}$. Therefore, the minimal values of $U_{\beta, F}$ are referred to as "zero-temperature case", or the ground states. It was proved in [16] that there are 4 different phases of the ground states for $U_{\beta, F}$ depending on $F$.

2.2. Results. We prove here that at any positive temperature there are 5 different phases; these are listed in the following theorem in the increasing order of external force.

We use the notation $g(N)=\Theta(h(N))$ if both hold: $g(N)=O(h(N))$ and $h(N)=O(g(N))$.

THEOREM 2.2. Let $t>0$ and $\beta>0$ be fixed arbitrarily, and let

$$
\bar{Y}=\left(Y_{0}=0, Y_{1}, \ldots, Y_{N-1}, Y_{N}=1\right)
$$


be a random vector in $\mathcal{S}=\left\{\left(y_{0}, \ldots, y_{N}\right): 0=y_{0}<\cdots<y_{N}=1\right\}$ with the Gibbs density

$$
f_{\bar{Y}}(\bar{y})=\frac{e^{-\frac{1}{t} U_{\beta, F}(\bar{y})}}{Z_{\frac{1}{t} U_{\beta, F}}(N)}, \quad \bar{y} \in \mathcal{S},
$$

where the potential function $U_{\beta, F}$ is given by (2.3).

Define

$$
F_{c r}(N)=4 \beta N \text {. }
$$

For any fixed $t>0$ and $\beta>0$, the following holds when $N \rightarrow \infty$.

(a) Weak subcritical force. If $F(N)=o(N)$, then for all $1 \leq k \leq N$,

(2.7) $\mathbb{E}\left(Y_{N+1-k}-Y_{N-k}\right)=\frac{1}{N}\left(1-\frac{F}{2 \beta N}\left(\frac{k}{N}-\frac{1}{2}\right)+O\left(\frac{F^{2}}{N^{2}}\right)+O\left(\frac{\log N}{\sqrt{N}}\right)\right)$

and

$$
\operatorname{Var}\left(Y_{k}-Y_{k-1}\right)=\frac{t}{2 \beta N^{3}}(1+o(1)) .
$$

(b) Subcritical force. If $F(N)=F_{0} N<F_{c r}(N)$, that is,

$$
F_{0}<4 \beta \text {, }
$$

then

$$
\mathbb{E}\left(Y_{N+1-k}-Y_{N-k}\right)=\frac{1}{a_{k} N}\left(1+O\left(\frac{\log N}{\sqrt{N}}\right)\right)
$$

and

$$
\operatorname{Var}\left(Y_{N+1-k}-Y_{N-k}\right)=\frac{t}{2 \beta a_{k}^{3} N^{3}}(1+o(1)),
$$

where

$$
a_{k}=\sqrt{1+\left(\frac{k}{N}-\frac{1}{2}\right) \frac{F_{0}}{\beta}+\frac{F_{0}^{2}}{16 \beta^{2}}} .
$$

(c) Critical force. If $F(N)=F_{c r}(N)=4 \beta N$, then

$$
\mathbb{E}\left(Y_{N+1-k}-Y_{N-k}\right)=\sqrt{\frac{1}{4(k-1) N+|\Theta(N)|}}+\frac{1}{k^{3 / 4}} O\left(\frac{\log N}{N^{3 / 4}}\right),
$$

and

$$
\operatorname{Var}\left(Y_{k}-Y_{k-1}\right)=\frac{1}{k^{3 / 4}} O\left(\frac{(\log N)^{2}}{N^{3 / 4}}\right)
$$


(d) Supercritical force. If $F(N)=F_{0} N>F_{c r}(N)$, that is, $F_{0}>4 \beta$, then

$$
1-\mathbb{E} Y_{N-1}=\mathbb{E}\left(Y_{N}-Y_{N-1}\right)=1-\sqrt{\frac{4 \beta}{F_{0}}}+O\left(\frac{1}{\sqrt{N}}\right)
$$

and

$$
\operatorname{Var}\left(Y_{N-1}\right)=\operatorname{Var}\left(Y_{N}-Y_{N-1}\right)=O\left(\frac{1}{\sqrt{N}}\right),
$$

while for all $k \geq 2$

$$
\mathbb{E}\left(Y_{N+1-k}-Y_{N-k}\right)=\sqrt{\frac{\beta}{(k-1) F_{0} N+t \lambda_{0}}}+\frac{1}{k^{3 / 4}} O\left(\frac{\log N}{N^{3 / 4}}\right),
$$

and

$$
\operatorname{Var}\left(Y_{N+1-k}-Y_{N-k}\right)=\frac{1}{k^{3 / 2}} O\left(\frac{(\log N)^{2}}{N^{3 / 2}}\right),
$$

where $\lambda_{0}=\lambda_{0}\left(\beta, F_{0}, t\right)$ is the unique solution to

$$
\frac{\int_{0}^{1} x e^{\lambda_{0} x-\frac{\beta}{t x}} d x}{\int_{0}^{1} e^{\lambda_{0} x-\frac{\beta}{t x}} d x}=1-\sqrt{\frac{4 \beta}{F_{0}}} .
$$

(e) Strong supercritical force. If $F(N) \gg N$, then

$$
1-\mathbb{E} Y_{N-1}=\mathbb{E}\left(Y_{N}-Y_{N-1}\right)=1-O\left(\sqrt{\frac{N}{F}}+N^{-3 / 2}\right),
$$

and

$$
\operatorname{Var}\left(Y_{N-1}\right)=O\left(\frac{N}{F}+N^{-3 / 2}\right) .
$$

In all statements the terms $O, o$ and $\Theta$ are uniform in $k$, but may depend on $t$ and $\beta$.

This theorem confirms that the phase transitions discovered in [16] for the ground states, take place for the Gibbs measure at any positive finite temperature as well. Remarkably, the principal terms for the asymptotics of the mean interspaces do not depend on temperature $t$ in the subcritical phases (a) and (b), and moreover they are equal to their counterparts for the ground states described in [16]. This suggests the continuity of the expectation of the inter-spaces at $t=0$ in the subcritical phase. Note that in [16] the ground states for the cases (b) and (c) are described as one phase. Here, at positive temperature, we see that there are different scalings under conditions (b) and (c), respectively.

Figure 1 helps one to visualize the positions of the particles in different phases. The particles at each phase are placed at intervals equal to the principal terms of 
a)

b)

$$
\text { c) }
$$

d)

e)

FIG. 1. $n=100, \beta=1$, (a) $F=1$, (b) $F_{0}=1$, (c) $\Theta(N)=N$, (d) $F_{0}=16$, (e) $F=N^{3}$.

mean values found in Theorem 2.2. Five different phases described by Theorem 2.2 are the following.

(a) Weak subcritical force. The particles remain to be equally spaced on the average, at distance $1 / N$, just as for $F=0$, and the variances are of order $N^{-3}$.

(b) Subcritical force. The same orders $N^{-1}$ and $N^{-3}$ for the mean and the variance, respectively, of the inter-spaces are preserved, however, they are not homogeneous any longer. The constants $a_{k}$ take different values between $a_{1}=$ $\frac{1}{1-\frac{F_{0}}{4 \beta}}(1+o(1 / N))>1$ and $\frac{1}{2}<a_{N}=\frac{1}{1+\frac{F_{0}}{4 \beta}}<1$. In particular, when $F_{0} \uparrow 4 \beta$ one has $a_{N} \rightarrow 1 / 2$, while $a_{1} \rightarrow \infty$.

(c) Critical force. All the inter-spaces converge to zero (in $L^{2}$ at least). Hence, the particles still densely cover the entire interval; however, the order of the mean varies from $N^{-1 / 2}$ to $N^{-1}$.

(d) Supercritical force. The particles occupy densely only a positive fraction of the interval $[0,1]$, namely $\left(0,1-\sqrt{\frac{4 \beta}{F_{0}}}\right)$, where the scaling varies as in (c), while on the remaining part there are no particles (except the ones with fixed positions at the ends).

(e) Strong supercritical force. All the particles (except the ones with fixed positions at the ends) are jammed towards one end.

The clear analogy allows one to speculate that this model should be versatile in physics for explaining phenomena of superconductivity.

We are able to define the precise asymptotics for the variance only in the cases of weak external force. Notice also that in the weak force case we use even sharper results, as asymptotics for the densities (see proofs in Section 3.5). Our proof does not show whether the upper bounds in the cases (c), (d), (e) are optimal. (One may guess that the optimal bounds will coincide with the ones derived in Corollary 3.8 below.) Still due to the very small variance one can even get further information on the macro-characteristics of the systems as, for example, $Y_{m+k}-Y_{m}$ for any $0 \leq$ 
$m<m+k \leq N$. At least in all cases except the critical one, our results still yield a concentration of these characteristics around their mean. As a side result, we also mention (Remark 3.12 below) that at least in the subcritical case our analysis yields exact asymptotic for the partition function, and thus the phase transition might be observed in the macro-states as well.

2.3. Possible generalizations. The analysis here relies on the specific assumption that the particles are hardly aligned so that only the nearest-neighbours interactions are counted, the assumption which makes the model "exactly solvable". However, it certainly suggests that a similar result should hold as well when the $k$ neighbours interactions are counted, or even all pairwise interactions are counted. The case of finite $k$ should qualitatively be exactly same as the one treated here: up to constants the scaling limits should be the same.

Further conjecture could be the following: when all the interactions are taken into account the critical value for the external force will be of order $N \log N$. When the external force is at most $o(N \log N)$, the spacings will be of nonuniform over the interval order

$$
\frac{\log N}{N \log \left(\frac{N}{2}-k+2\right)}, \quad k \leq N / 2 \quad \text { and } \quad \frac{\log N}{N \log \left(k-\frac{N}{2}+2\right)}, \quad k>N / 2
$$

(compare to Theorem 2.2 where the spacings in presence of weak force are uniform). Then increase of the external constant force will result again in a number of different phases, which are not monotone, unlike the present case (Theorem 2.2 states that the particles' density is monotone increasing towards one end). However, qualitatively, we expect again a finite number (5?) of different phases.

Another direction of the generalization of the model is the form of the external force. First, it should be noted that already in [16] this question was discussed for the ground (zero temperature) case. It was proved there that if the external force is monotone increasing (i.e., replace $F_{s}=F$ in the Assumption 2.1 by $F_{s}=F h(s)$, where $h(s)$ is positive, monotone and bounded on $[0,1])$, then there is a unique equilibrium ground state. This should be also the case for the Gibbs states. One can work out this model using the same arguments as here. Our conjecture is that the phase transitions along $F=F(N)$ will be qualitatively the same as in case $h(s) \equiv 1$ considered here.

However, the picture will be different when the force is nonmonotone over the interval. As it was exemplified in [16], in this case one may expect nonuniqueness of the minimums of the energy function. This should result in another phase transition at low temperature.

The analysis presented here is not much sensitive to the form of the potential $V(x)$; in fact different cases were already treated in [17]. The scaling of the critical external force will be different of course, but the entire program can be worked out along the same lines (see also [17] for the systems without external force). Here, 
we focus on the Coulomb potential because the aim of our model is to explain the physics of the electric current.

Finally, we mention that the models on graphs with different from the interval structure have not yet been studied, but they might bring new features.

\section{Proof of Theorem 2.2.}

3.1. Scaling along the temperature $t$. Observe that the function of potential $U_{\beta, F}$ in (2.3) has the following scaling property: for any $\beta, F$ and $t>0$,

$$
\frac{1}{t} U_{\beta, F}(\bar{y})=U_{\frac{\beta}{t}, \frac{F}{t}}(\bar{y}) .
$$

Correspondingly, for any $\beta, F$ and $t>0$ the density function $f_{\bar{Y}}$ in (2.6) can be written as

$$
f_{\bar{Y}}(\bar{y})=\frac{e^{-\frac{1}{t} U_{\beta, F}(\bar{y})}}{Z_{\frac{1}{t} U_{\beta, F}}(N)}=\frac{e^{-U_{\hat{\beta}, \hat{F}}(\bar{y})}}{Z_{U_{\hat{\beta}, \hat{F}}}(N)},
$$

where $\hat{\beta}=\frac{\beta}{t}$, and $\hat{F}=\frac{F}{t}$.

Therefore, with a slight abuse of notation, namely writing $\beta$ and $F$ instead of $\hat{\beta}$ and $\hat{F}$, we shall first study $\bar{Y}$ with the density

$$
f_{\bar{Y}}(\bar{y})=\frac{e^{-U_{\beta, F}(\bar{y})}}{Z_{U_{\beta, F}(N)}}, \quad \bar{y} \in \mathcal{S},
$$

and then in the resulting formulas we shall transform the parameters back by

$$
\beta \rightarrow \frac{\beta}{t}, \quad F \rightarrow \frac{F}{t} .
$$

3.2. Representation of vector of inter-spaces. Consider $\bar{Y}$ with the density (3.3). We begin with establishing a useful representation for the random variables $Y_{k}-Y_{k-1}, 1 \leq k \leq N$.

Proposition 3.1. Let $X_{k}, k=1, \ldots, N$, be independent random variables with density functions

$$
f_{X_{k}}(x)=\frac{1}{c_{k}} e^{-\beta V(x)-F k x}=\frac{1}{c_{k}} e^{-\frac{\beta}{x}-F k x}, \quad x \in[0,1],
$$

where

$$
c_{k}=\int_{0}^{1} e^{-\frac{\beta}{x}-F k x} d x .
$$

Then the following distributional identity holds for all $N \geq 2$ :

$$
\left.\left(1-Y_{N-1}, Y_{N-1}-Y_{N-2}, \ldots, Y_{2}-Y_{1}, Y_{1}\right) \stackrel{d}{=}\left(X_{1}, \ldots, X_{N}\right)\right|_{\sum_{i=1}^{N} X_{i}=1} .
$$


PROOF. Using Assumptions 2.1, let us rewrite the potential function as follows:

$$
\begin{aligned}
U(\bar{y}) & =U_{\beta, F}(\bar{y})=\beta \sum_{k=1}^{N} V\left(y_{k}-y_{k-1}\right)+F \sum_{k=1}^{N}(N-k+1)\left(y_{k}-y_{k-1}\right) \\
& =\beta \sum_{k=1}^{N} V\left(y_{N+1-k}-y_{N-k}\right)+F \sum_{k=1}^{N} k\left(y_{N+1-k}-y_{N-k}\right),
\end{aligned}
$$

where $y_{0}=1, y_{N}=1$. Denote here for all $1 \leq k \leq N$ and $x \in(0,1]$

$$
g_{k}(x)=\beta V(x)+F k x=\frac{\beta}{x}+F k x .
$$

With this notation

$$
U(\bar{y})=\sum_{k=1}^{N} g_{k}\left(y_{N+1-k}-y_{N-k}\right),
$$

and then the density of $Y_{1}, \ldots, Y_{N-1}$ given by (3.3) can be rewritten as

$$
f_{Y_{1}, \ldots, Y_{N-1}}\left(y_{1}, \ldots, y_{N-1}\right)=\frac{1}{Z_{U_{\beta, F}}(N)} e^{-\sum_{k=1}^{N} g_{k}\left(y_{N+1-k}-y_{N-k}\right)} .
$$

From here, we derive the joint density of the increments $1-Y_{N-1}, Y_{N-1}-$ $Y_{N-2}, \ldots, Y_{2}-Y_{1}$ :

$$
f_{1-Y_{N-1}, \ldots, Y_{2}-Y_{1}}\left(x_{1}, \ldots, x_{N-1}\right)=\frac{1}{Z_{U_{\beta, F}}(N)} e^{-\sum_{k=1}^{N-1} g_{k}\left(x_{k}\right)-g_{N}\left(1-\sum_{k=1}^{N-1} x_{k}\right)}
$$

for all positive values $x_{1}, \ldots, x_{N-1}$, such that $\sum_{k=1}^{N-1} x_{k} \leq 1$.

On the other hand, straight from the definition of the variables $X_{k}$, we get

$$
\begin{aligned}
f_{X_{1}, \ldots, X_{N-1} \mid \sum_{k=1}^{N} X_{k}=1}\left(x_{1}, \ldots, x_{N-1}\right) \\
=\left\{\begin{array}{c}
\frac{\left(\prod_{k=1}^{N} c_{k}\right)^{-1} e^{-\sum_{k=1}^{N-1} g_{k}\left(x_{k}\right)-g_{N}\left(1-\sum_{k=1}^{N-1} x_{k}\right)}}{f_{\sum_{k=1}^{N} X_{k}}(1)} \\
\text { if } x_{k}>0 \forall 1 \leq k \leq N-1, \text { and } \sum_{k=1}^{N-1} x_{k} \leq 1, \\
\text { otherwise. }
\end{array}\right.
\end{aligned}
$$

Since we have the same functions on the right in (3.11) and (3.10), it follows that

$$
\left.\left(1-Y_{N-1}, \ldots, Y_{2}-Y_{1}\right) \stackrel{d}{=}\left(X_{1}, \ldots, X_{N-1}\right)\right|_{\sum_{i=1}^{N} X_{i}=1} .
$$

Finally, we observe that $Y_{1}=1-\left(1-Y_{N-1}\right)-\cdots-\left(Y_{2}-Y_{1}\right)$, while conditionally on $\sum_{i=1}^{N} X_{i}=1$ one has $X_{N}=1-\sum_{i=1}^{N-1} X_{i}$. Therefore, the statement of the proposition follows by (3.12). 
Let us mention here for a further reference, that as a direct consequence of the equality of (3.11) and (3.10) we have a useful representation for the partition function as well, namely

$$
Z_{U_{\beta, F}}(N)=\left(\prod_{k=1}^{N} c_{k}\right) f_{\sum_{k=1}^{N} X_{k}}(1) .
$$

Proposition 3.1 implies immediately the following corollary.

COROLLARY 3.2. For all $1 \leq k \leq N$, the following identity in distribution holds:

$$
Y_{N-k+1}-\left.Y_{N-k} \stackrel{d}{=} X_{k}\right|_{\sum_{i=1}^{N} X_{i}=1},
$$

where $Y_{N}=1$ and $Y_{0}=0$.

Therefore, in order to prove Theorem 2.2 we shall study the conditional density of $X_{k}$ given $\sum_{i=1}^{N} X_{i}=1$.

3.3. Lagrange multiplier. Here, we shall solve some optimization problem, introducing an auxiliary parameter, and hence, introducing new distributions.

Following the ideas of [7], let us embed the distribution of $X_{k}$ (defined in Proposition 3.1) into a more general class. Namely, for any $\lambda \in \mathbb{R}$ define a density function

$$
f_{k, \lambda}(u)=\frac{1}{c_{k}(\lambda)} e^{-\frac{\beta}{u}-(\lambda+F k) u}, \quad u \in[0,1],
$$

where

$$
c_{k}(\lambda)=\int_{0}^{1} e^{-\frac{\beta}{u}-(\lambda+F k) u} d u,
$$

and denote the corresponding random variable $X_{k, \lambda}$. In these notation, $X_{k} \stackrel{d}{=} X_{k, 0}$. We assume that for each fixed $\lambda$ the random variables $X_{k, \lambda}, k=1, \ldots, N$, are independent. The remarkable property of these random variables is that for any $\lambda \in \mathbb{R}$ the following equality in distribution holds:

$$
\left.\left.X_{k}\right|_{\sum_{i=1}^{N} X_{i}=1} \stackrel{d}{=} X_{k, \lambda}\right|_{\sum_{i=1}^{N} X_{i, \lambda}=1} .
$$

Indeed, denoting

$$
S_{N}=\sum_{i=1}^{N} X_{i}, \quad S_{N, \hat{k}}=\sum_{i \neq k} X_{i}
$$

and, correspondingly,

$$
S_{N, \lambda}=\sum_{i=1}^{N} X_{i, \lambda}, \quad S_{N, \hat{k}, \lambda}=\sum_{i \neq k} X_{i, \lambda},
$$


it is straightforward to check that for the conditional densities one has

$$
f_{X_{k} \mid \sum_{i=1}^{N} X_{i}=1}(x):=\frac{f_{X_{k}}(x) f_{S_{N, \hat{k}}}(1-x)}{f_{S_{N}}(1)}=\frac{f_{k, \lambda}(x) f_{S_{N, \hat{k}, \lambda}}(1-x)}{f_{S_{N, \lambda}}(1)},
$$

that is, the right-hand side does not depend on $\lambda$.

Diaconis and Freedman [7] used this property to prove the conditional central limit theorem for the identically distributed random variables; their result was then used in [17] to treat the case without external force (which is still the i.i.d. case ).

Here, we show that a similar argument works even without the assumption of the identity of distributions. The main idea is to tune the free parameter $\lambda$ so that the condition $\sum_{k=1}^{N} X_{k, \lambda}=1$ becomes a natural one in the following sense. Since we have a sum of independent random variables, we may expect that due to the central limit theorem the density of the normalized sum $S_{N, \lambda}$ converges to the density of a normal distribution with the maximum at the point of its expected value. Therefore, we shall choose $\lambda$ so that

$$
\mathbb{E} S_{N, \lambda}=\sum_{k=1}^{N} \mathbb{E} X_{k, \lambda}=1
$$

Before solving (3.18), let us note that with a help of random variables $X_{k, \lambda}$ we can rewrite also the representation for the partition function (3.13) as follows:

$$
Z_{U_{\beta, F}}(N)=e^{\lambda}\left(\prod_{k=1}^{N} c_{k}(\lambda)\right) f_{\sum_{k=1}^{N} X_{k, \lambda}}(1) .
$$

Now we turn to (3.18). Notice that the definition (3.15) yields (see also [17] and [7]) that $\mathbb{E} X_{k, \lambda}$ is a strictly decreasing function of $\lambda$, such that $\mathbb{E} X_{k, \lambda} \rightarrow 0$ when $\lambda \rightarrow+\infty$ while $\mathbb{E} X_{k, \lambda} \rightarrow 1$ when $\lambda \rightarrow-\infty$. Hence, equation (3.18) defines uniquely $\lambda$ which satisfies this condition. To solve equation (3.18), first we consider $\mathbb{E} X_{k, \lambda}$. Notice, that in [17] one can find the principal term of the asymptotic of this value. Here, using the arguments of [17], we get more details.

Proposition 3.3. For any $1 \leq k \leq N$ and $F \geq 0$,

$$
m_{k, \lambda}:=\mathbb{E} X_{k, \lambda}=\sqrt{\frac{\beta}{k F+\lambda}}+\frac{3}{4(k F+\lambda)}(1+o(1)),
$$

and

$$
\operatorname{Var}\left(X_{k, \lambda}\right)=\frac{\sqrt{\beta}}{2(k F+\lambda)^{3 / 2}}(1+o(1))
$$

as $k F+\lambda \rightarrow \infty$. 
PROOF. Let us write here

$$
\lambda_{k}=\lambda+k F
$$

and, correspondingly, $X_{k, \lambda}=X_{\lambda_{k}}$ and $c_{k}(\lambda)=c\left(\lambda_{k}\right)$ (these notation are consistent with the ones in [17]).

For any $\lambda>0$ and $\alpha \in\{1,2,3\}$, define

$$
I_{\alpha}(\lambda, \beta)=\int_{0}^{\infty} x^{\alpha-1} e^{-\lambda x-\frac{\beta}{x}} d x
$$

Then we have

$$
\mathbb{E} X_{\lambda}=\frac{\int_{0}^{1} x e^{-\lambda x-\frac{\beta}{x}} d x}{c(\lambda)}=\frac{\int_{0}^{1} x e^{-\lambda x-\frac{\beta}{x}} d x}{\int_{0}^{1} e^{-\lambda x-\frac{\beta}{x}} d x}=\frac{I_{2}(\lambda, \beta)+O\left(e^{-\frac{1}{2} \lambda}\right)}{I_{1}(\lambda, \beta)+O\left(e^{-\frac{1}{2} \lambda}\right)},
$$

as $\lambda \rightarrow \infty$. It was observed in [17] that

$$
I_{\alpha}(\lambda, \beta)=\frac{2 \beta^{\alpha / 2} K_{\alpha}(2 \sqrt{\lambda \beta})}{\lambda^{\alpha / 2}},
$$

where $K_{\alpha}(z)$ is a Bessel function for which the asymptotic expansion when $z \rightarrow \infty$ is known to be

$$
K_{\alpha}(z)=\sqrt{\frac{\pi}{2}} \frac{e^{-z}}{\sqrt{z}}\left(1+\frac{4 \alpha^{2}-1}{8 z}+o\left(z^{-1}\right)\right)
$$

(consult, e.g., [9]).

REMARK 3.4. Asymptotic (3.26) holds as well when $z \rightarrow \infty$ in a sector of a complex plain with $|\arg z| \leq \frac{\pi}{2}-\varepsilon$ if $0<\varepsilon<\frac{\pi}{2}$.

Hence, using (3.25) and then (3.26), we derive from (3.24)

$$
\begin{aligned}
\mathbb{E} X_{\lambda} & =\frac{\frac{\beta}{\lambda} K_{2}(2 \sqrt{\lambda \beta})+O\left(e^{-\frac{1}{2} \lambda}\right)}{\sqrt{\frac{\beta}{\lambda}} K_{1}(2 \sqrt{\lambda \beta})+O\left(e^{-\frac{1}{2} \lambda}\right)} \\
& =\sqrt{\frac{\beta}{\lambda}}\left(1+\frac{3}{4 \sqrt{\lambda \beta}}+o\left(\lambda^{-1 / 2}\right)\right)=\sqrt{\frac{\beta}{\lambda}}+\frac{3}{4 \lambda}+o\left(\lambda^{-1}\right),
\end{aligned}
$$

which together with (3.22) yields (3.20). For a further reference, let us state separately

$$
c(\lambda)=2 \sqrt{\frac{\beta}{\lambda}} K_{1}(2 \sqrt{\lambda \beta})+O\left(e^{-\frac{1}{2} \lambda}\right)=\frac{\sqrt{\pi} \beta^{1 / 4}}{\lambda^{3 / 4}} e^{-2 \sqrt{\lambda \beta}}\left(1+O\left(\lambda^{-1 / 2}\right)\right) .
$$


In a similar manner, we derive as well

$$
\begin{aligned}
\mathbb{E} X_{\lambda}^{2} & =\frac{\int_{0}^{1} x^{2} e^{-\lambda x-\frac{\beta}{x}} d x}{\int_{0}^{1} e^{-\lambda x-\frac{\beta}{x}} d x}=\frac{I_{3}(\lambda)+O\left(e^{-\frac{1}{2} \lambda}\right)}{I_{1}(\lambda)+O\left(e^{-\frac{1}{2} \lambda}\right)} \\
& =\frac{\beta}{\lambda}\left(1+\frac{2}{\sqrt{\lambda \beta}}+o\left(\lambda^{-1 / 2}\right)\right),
\end{aligned}
$$

which together with (3.27) yields

$$
\operatorname{Var}\left(X_{\lambda}\right)=\frac{\sqrt{\beta}}{2 \lambda^{3 / 2}}(1+o(1)) .
$$

This together with (3.22) implies (3.21). The proposition is proved.

Next, we describe the asymptotic of $\lambda=\lambda(N, F)$ which solves (3.18).

In the following, we shall use "weak force" for both conditions "weak subcritical force" and "subcritical force", while "strong force" will be used for both "supercritical force" and "strong supercritical force".

LEMMA 3.5. Assume that $\lambda=\lambda(N, F)$ is chosen so that (3.18) holds, that is,

$$
\sum_{k=1}^{N} \mathbb{E} X_{k, \lambda}=1
$$

Then the following statements take place:

(a) Weak force. If $0 \leq F<4 \beta N$, then

$$
\lambda(N, F)=\beta\left(1-\frac{F}{4 \beta N}\right)^{2} N^{2}+O(N) .
$$

(b) Critical force. If $F=4 \beta N$, then $\lambda(N, F)<0$, and for some positive a

$$
4 \beta N+\lambda(N, F) \geq a N .
$$

(c) Strong force. If $F=F_{0} N$, where $F_{0}>4 \beta$, including $F_{0}=F_{0}(N) \gg 1$, then

$$
\lambda(N, F)=-F-\lambda_{0}+o\left(\frac{1}{\sqrt{F}}\right),
$$

where $\lambda_{0}=\lambda_{0}\left(\beta, F_{0}\right)$ is the unique solution to

$$
\frac{\int_{0}^{1} x e^{\lambda_{0} x-\frac{\beta}{x}} d x}{\int_{0}^{1} e^{\lambda_{0} x-\frac{\beta}{x}} d x}=1-\sqrt{\frac{4 \beta}{F_{0}}} .
$$

REMARK 3.6. In the case (c) function $\lambda_{0}=\lambda_{0}\left(F_{0}\right)$ is increasing in $F_{0}$; in particular, $\lambda_{0}\left(F_{0}\right) \rightarrow+\infty$ as $F_{0} \rightarrow \infty$, but so that $\lambda_{0}\left(F_{0}\right)=\Theta\left(F_{0}^{1 / 2}\right)$. 
PROOF. Let us solve (3.30) when $F \geq 0$. Recall that we denote here $X_{k, \lambda}=$ $X_{\lambda_{k}}$, where $\lambda_{k}=k F+\lambda$. Observe that for any $k$ and $\lambda$ the value $\mathbb{E} X_{k, \lambda}$ [see formula (3.24)] is positive, and it is decreasing in $\lambda_{k}$, hence, in all parameters, $k, F$ and $\lambda$. Therefore, we have

$$
\sum_{k=1}^{N} \mathbb{E} X_{k, \lambda} \geq N \mathbb{E} X_{N, \lambda}
$$

Assuming equation (3.30) holds, this implies

$$
1=\sum_{k=1}^{N} \mathbb{E} X_{k, \lambda} \geq N \mathbb{E} X_{N, \lambda} .
$$

Hence, if $\lambda$ satisfies (3.30), and thus (3.35), we must have $\mathbb{E} X_{N, \lambda} \rightarrow 0$, and thus

$$
N F+\lambda \rightarrow \infty \text {. }
$$

Using formula (3.20), we derive from (3.35)

$$
1=\sum_{k=1}^{N} \mathbb{E} X_{k, \lambda} \geq N\left(\sqrt{\frac{\beta}{N F+\lambda}}+\frac{3}{4(N F+\lambda)}(1+o(1))\right),
$$

where $o(1) \rightarrow 0$ as $N F+\lambda \rightarrow \infty$.

Consider now separately different cases. We start with case (a) Weak force.

(a.1) Weak subcritical force. The bound in (3.37) tells us that if $F=o(N)$ and $\lambda$ satisfies (3.18) then for some positive $a$

$$
\lambda \geq a N^{2},
$$

and, therefore, for all $F \geq 0$ and all $k \geq 1$

$$
\lambda_{k}=k F+\lambda \geq a N^{2} \rightarrow \infty \text {. }
$$

Having this uniform in $k$ bound, we can use (3.20) to derive

$$
\sum_{k=1}^{N} \mathbb{E} X_{k, \lambda}=\sum_{k=1}^{N}\left(\sqrt{\frac{\beta}{k F+\lambda}}+\frac{3}{4(k F+\lambda)}(1+o(1))\right),
$$

where $o(1) \rightarrow 0$ as $N \rightarrow \infty$ uniformly in $k$ due to (3.39). Hence, solving (3.30) when $F=o(N)$ is equivalent to finding the (unique) solution to

$$
1=\sum_{k=1}^{N}\left(\sqrt{\frac{\beta}{k F+\lambda}}+\frac{3}{4(k F+\lambda)}(1+o(1))\right) .
$$

As we argued above, if $F=o(N)$ the solution to this equation satisfies (3.38) and, therefore, equation (3.41) yields

$$
1=\sum_{k=1}^{N} \sqrt{\frac{\beta}{k F+\lambda}}+O\left(\frac{1}{N}\right) .
$$


First, we note that when $F=0$ (this case was considered in [17]) we get immediately from (3.42) that

$$
\lambda(N, 0)=\beta N^{2}(1+O(1 / N)) .
$$

For any $0<F=o(N)$, we derive from equation (3.42) and bound (3.38)

$$
\begin{aligned}
1 & =\sqrt{\frac{\beta}{F}}\left(\int_{1}^{N} \frac{1}{\sqrt{x+\frac{\lambda}{F}}} d x+O\left(\frac{1}{\sqrt{1+\frac{\lambda}{F}}}\right)\right)+O\left(\frac{1}{N}\right) \\
& =2 \sqrt{\beta} \frac{N-1}{\sqrt{F N+\lambda}+\sqrt{F+\lambda}}+O\left(\frac{1}{N}\right) .
\end{aligned}
$$

It is straightforward to obtain the solution to the last equation [where $F=o(N)$ ]:

$$
\lambda(N, F)=\beta N^{2}\left(1-\frac{F}{4 \beta N}\right)^{2}+O(N) .
$$

(a.2) Subcritical force. Assume now that $F=F_{0} N$ for some positive $F_{0}$. Taking into account (3.20), first we derive for all $\lambda \geq 0$

$$
\sum_{k=1}^{N} \mathbb{E} X_{k, \lambda}=\sum_{k=1}^{N}\left(\sqrt{\frac{\beta}{k F+\lambda}}+\frac{3}{4(k F+\lambda)}(1+o(1))\right),
$$

where $o(1) \rightarrow 0$ as $N \rightarrow \infty$ uniformly in $k$ due to the uniform bound

$$
k F+\lambda \geq F_{0} N \text {. }
$$

Using the fact that $F=F_{0} N$, we get from (3.46)

$$
\sum_{k=1}^{N} \mathbb{E} X_{k, \lambda}=\sum_{k=1}^{N} \sqrt{\frac{\beta}{k F_{0} N+\lambda}}+\min \left\{\left|O\left(\frac{\log N}{N}\right)\right|,\left|O\left(\frac{N}{\lambda}\right)\right|\right\},
$$

from where similar to (3.44) we obtain

$$
\begin{aligned}
\sum_{k=1}^{N} \mathbb{E} X_{k, \lambda} & =\sqrt{\frac{\beta}{F_{0} N}}\left(\int_{1}^{N} \frac{1}{\sqrt{x+\frac{\lambda}{F_{0} N}}} d x+O\left(\frac{1}{\sqrt{1+\frac{\lambda}{F_{0} N}}}\right)\right)+\mathcal{R}(N) \\
& =2 \sqrt{\beta} \frac{N-1}{\sqrt{F_{0} N^{2}+\lambda}+\sqrt{F_{0} N+\lambda}}+O\left(\frac{1}{\sqrt{F_{0} N+\lambda}}\right)+\mathcal{R}(N),
\end{aligned}
$$

where we denoted

$$
\mathcal{R}(N)=\min \left\{\left|O\left(\frac{\log N}{N}\right)\right|,\left|O\left(\frac{N}{\lambda}\right)\right|\right\}=o(1) .
$$

Observe that the principal term (when $N \rightarrow \infty$ ) on the right in (3.48) decays both in $F_{0}$ and in $\lambda$; in particular, when $\lambda=0$ it equals to

$$
\sqrt{\frac{4 \beta}{F_{0}}}
$$


This yields that when $\frac{4 \beta}{F_{0}}<1$ there is no positive solution $\lambda$ to (3.30). On the other hand, if $\frac{4 \beta}{F_{0}}>1$ then the solution $\lambda$ to (3.30) is positive, and moreover by (3.48), it must be of order $N^{2}$. Indeed, under the assumption that $\lambda / N^{2}$ is strictly positive, we have $\mathcal{R}(N)=O\left(N^{-1}\right)$ and then the equations (3.30) and (3.48) give us

$$
1=2 \sqrt{\beta} \frac{N}{\sqrt{F_{0} N^{2}+\lambda}+\sqrt{F_{0} N+\lambda}}\left(1+O\left(\frac{1}{N}\right)\right) .
$$

Solving this we derive the solution $\lambda$ to (3.50),

$$
\lambda(N, F)=\beta N^{2}\left(1-\frac{F_{0}}{4 \beta}\right)^{2}+O(N),
$$

which is positive and of order $N^{2}$ when $F_{0}<4 \beta$. Due to the uniqueness, this is also the solution to (3.30) when $F_{0}<4 \beta$. This together with (3.45) and (3.43) confirms statement (a) of the lemma.

(b) Critical force. When $F=4 \beta N$, the principal term on the right in (3.48) for any $\lambda \geq 0$ equals 1 . Notice also that by (3.46) in this case we have for any $\lambda \geq 0$

$$
\begin{aligned}
\sum_{k=1}^{N} \mathbb{E} X_{k, \lambda} & \leq \sum_{k=1}^{N} \mathbb{E} X_{k, 0}=\sum_{k=1}^{N}\left(\sqrt{\frac{\beta}{k 4 \beta N}}+\frac{3}{4 k(4 \beta N)}(1+o(1))\right) \\
& =\frac{1}{2 \sqrt{N}} \sum_{k=1}^{N} \frac{1}{\sqrt{k}}+O\left(\frac{\log N}{N}\right) \\
& <\frac{1}{2 \sqrt{N}}\left(1+\int_{2}^{N+1} \frac{1}{\sqrt{x-1}} d x\right)+O\left(\frac{\log N}{N}\right) \\
& =1-\frac{1}{2 \sqrt{N}}+O\left(\frac{\log N}{N}\right)<1
\end{aligned}
$$

for all large $N$. Hence, the solution $\lambda$ to (3.30) when $F=4 \beta N$ is negative.

Assume, $\lambda<0$. Then as in (3.52),

$$
\sum_{k=2}^{N} \mathbb{E} X_{k, \lambda} \geq \sum_{k=2}^{N} \mathbb{E} X_{k, 0} \geq \sum_{k=2}^{N} \sqrt{\frac{\beta}{k 4 \beta N}}=1-\left|O\left(\frac{1}{\sqrt{N}}\right)\right| .
$$

Therefore, if $\lambda$ is a solution to (3.30) when $F=4 \beta N$, it must be negative, and it should satisfy

$$
\mathbb{E} X_{1, \lambda}=O\left(\frac{1}{\sqrt{N}}\right) .
$$

By the formula (3.24), this means that $\lambda_{1}$ should satisfy

$$
\mathbb{E} X_{1, \lambda}=\frac{\int_{0}^{1} x e^{-\lambda_{1} x-\frac{\beta}{x}} d x}{\int_{0}^{1} e^{-\lambda_{1} x-\frac{\beta}{x}} d x}=O\left(\frac{1}{\sqrt{N}}\right),
$$


implying that $\lambda_{1}=F+\lambda \rightarrow+\infty$. This together with the asymptotic (3.20) yields that $\lambda$ satisfies (3.54) if and only if for some positive $a$ :

$$
F>F+\lambda=4 \beta N+\lambda \geq a N .
$$

Finally, using computations of (3.52) we check that if $\lambda<0$ and satisfies (3.55) then

$$
\begin{aligned}
\sum_{k=2}^{N} \mathbb{E} X_{k, \lambda} & =\sum_{k=2}^{N}\left(\sqrt{\frac{\beta}{k 4 \beta N+\lambda}}+\frac{3}{4 k(4 \beta N)}(1+o(1))\right) \\
& <\frac{1}{2 \sqrt{N}} \sum_{k=1}^{N} \frac{1}{\sqrt{k}}+O\left(\frac{\log N}{N}\right)<1-O\left(\frac{1}{\sqrt{N}}\right) .
\end{aligned}
$$

This together with (3.53) confirms both holds, (3.54) and

$$
\sum_{k=2}^{N} \mathbb{E} X_{k, \lambda}=1-O\left(\frac{1}{\sqrt{N}}\right)
$$

Therefore, the solution $\lambda$ to (3.30) is negative and satisfies (3.55). This proves statement (b) of the lemma.

(c) Strong force. To prove (c), we assume that $F=F_{0} N>4 \beta$. As we argued above, the (unique) solution $\lambda$ to (3.30) is negative in this case. Note first, that if

$$
-\frac{3}{2} F_{0} N<\lambda<0
$$

then uniformly in $k \geq 2$

$$
k F+\lambda \geq F_{0} N / 2 \rightarrow \infty .
$$

Therefore, for any $-\frac{3}{2} F_{0} N<\lambda<0$ we have similar to (3.40)

$$
\sum_{k=2}^{N} \mathbb{E} X_{k, \lambda}=\sum_{k=2}^{N}\left(\sqrt{\frac{\beta}{k F+\lambda}}+\frac{3}{4(k F+\lambda)}(1+o(1))\right),
$$

where $o(1)$ is uniform in $k \geq 2$, which yields

$$
\begin{aligned}
\sum_{k=2}^{N} \mathbb{E} X_{k, \lambda}= & 2 \sqrt{\beta} \frac{N-1}{\sqrt{F_{0} N^{2}+\lambda}+\sqrt{2 F_{0} N+\lambda}} \\
& +O\left(\frac{1}{\sqrt{2 F_{0} N+\lambda}}\right)+O\left(\frac{\log N}{F_{0} N}\right) \\
= & \sqrt{\frac{4 \beta}{F_{0}}}+O\left(\frac{1}{\sqrt{F_{0} N}}\right) .
\end{aligned}
$$


Hence, under assumption (3.56) equation (3.30) becomes

$$
1=\mathbb{E} X_{1, \lambda}+\sqrt{\frac{4 \beta}{F_{0}}}+O\left(\frac{1}{\sqrt{F_{0} N}}\right) .
$$

Set now

$$
\lambda=-F_{0} N-\lambda_{0}+O\left(\frac{1}{\sqrt{F_{0} N}}\right),
$$

where $\lambda_{0}$ satisfies (3.34). Then by the definition (3.15) and equation (3.34)

$$
\mathbb{E} X_{1, \lambda}=\frac{\int_{0}^{1} x e^{\lambda_{0} x-\frac{\beta}{x}+O\left(\frac{1}{\sqrt{F_{0} N}}\right) x} d x}{\int_{0}^{1} e^{\lambda_{0} x-\frac{\beta}{x}+O\left(\frac{1}{\sqrt{F_{0} N}}\right) x} d x}=1-\sqrt{\frac{4 \beta}{F_{0}}}+O\left(\frac{1}{\sqrt{F_{0} N}}\right),
$$

which yields as well the relation (3.59).

Supercritical force. When $F_{0}>4 \beta$ is a constant, then $\lambda_{0}$ is also of order constant and, therefore,

$$
0>\lambda=-F_{0} N-\lambda_{0}+O\left(\frac{1}{\sqrt{F_{0} N}}\right)>-\frac{3}{2} F_{0} N,
$$

which confirms (3.56).

Strong supercritical force. Consider $F_{0}=F_{0}(N) \rightarrow \infty$. Note that the function on the left-hand side in (3.61) is increasing in $\lambda_{0}$, and moreover

$$
0<1-\frac{\int_{0}^{1} x e^{\lambda_{0} x-\frac{\beta}{x}} d x}{\int_{0}^{1} e^{\lambda_{0} x-\frac{\beta}{x}} d x}=\frac{\int_{0}^{1}(1-x) e^{-\lambda_{0}(1-x)-\frac{\beta}{x}} d x}{\int_{0}^{1} e^{-\lambda_{0}(1-x)-\frac{\beta}{x}} d x}=\Theta\left(\lambda_{0}^{-1}\right)
$$

as $\lambda_{0} \rightarrow \infty$. This yields that solution $\lambda_{0}\left(F_{0}\right)$ to (3.61) for all large $F_{0}$ satisfies

$$
0<\lambda_{0}=\Theta\left(F_{0}^{1 / 2}\right)
$$

implying as well that $\lambda=-F_{0} N-\lambda_{0}+O\left(\frac{1}{\sqrt{F_{0} N}}\right)>-\frac{3}{2} F_{0} N$.

Hence, (3.60) yields statement (c) of the lemma. Lemma 3.5 and Remark 3.6 are proved.

REMARK 3.7. It follows from (3.61) that if $F_{0}=F_{0}(N)$ is unbounded, that is, when $F \gg N$, for the chosen $\lambda$ we have $X_{1, \lambda} \rightarrow 1$, while $\sum_{k=2}^{N} \mathbb{E} X_{k, \lambda} \rightarrow 0$ as $N \rightarrow \infty$.

COROLlaRY 3.8. Assume $\lambda=\lambda(N, F)$ satisfies (3.30), that is, it is chosen as in Lemma 3.5: 
(I) Weak force. If $\lim _{N \rightarrow \infty} F / N=F_{0}<4 \beta$, then there is a positive constant $C=C\left(\beta, F_{0}\right)$ such that

$$
\sigma_{N}^{2}=\sum_{k=1}^{N} \operatorname{Var}\left(X_{k, \lambda}\right)=\frac{C}{N^{2}}(1+o(1))
$$

where for any $k$

$$
\operatorname{Var}\left(X_{k, \lambda}\right)=\frac{\sqrt{\beta}}{2\left(k F+\beta\left(1-\frac{F_{0}}{4 \beta}\right)^{2} N^{2}\right)^{3 / 2}}(1+o(1))=O\left(N^{-3}\right) .
$$

(II) Critical force. If $\lim _{N \rightarrow \infty} F / N=4 \beta$, then

$$
\sum_{k=1}^{N} \operatorname{Var}\left(X_{k, \lambda}\right)=\Theta\left(N^{-3 / 2}\right)
$$

where for any $k$

$$
\operatorname{Var}\left(X_{k, \lambda}\right)=\frac{\sqrt{\beta}}{2(k F+\lambda)^{3 / 2}}(1+o(1)),
$$

where $\lambda$ satisfies (3.32).

(III) Strong force. If $\lim _{N \rightarrow \infty} F / N>4 \beta$, then there is a positive constant $C=$ $C(\beta)$ such that

$$
\sum_{k=2}^{N} \operatorname{Var}\left(X_{k, \lambda}\right)=\frac{C}{F^{3 / 2}}(1+o(1))=O\left(N^{-3 / 2}\right),
$$

where for any $k \geq 2$

$$
\operatorname{Var}\left(X_{k, \lambda}\right)=\frac{\sqrt{\beta}}{2(k-1)^{3 / 2} F^{3 / 2}}(1+o(1)),
$$

while

(a) supercritical force: if $\lim _{N \rightarrow \infty} F / N=F_{0}$ and $F_{0}>4 \beta$ is a positive constant, then

$$
\operatorname{Var}\left(X_{1, \lambda}\right)=\sigma\left(F_{0}\right)(1+o(1))
$$

where $\sigma\left(F_{0}\right)$ is also some positive constant;

(b) strong supercritical force: if $F \gg N$, then

$$
\operatorname{Var}\left(X_{1, \lambda}\right)=O\left(\left(\frac{N}{F}\right)^{3 / 4}\right)
$$


Proof. By (3.21), we have for $\lambda=\lambda(N, F)$

$$
\operatorname{Var}\left(X_{k, \lambda}\right)=\frac{\sqrt{\beta}}{2(k F+\lambda)^{3 / 2}}(1+o(1)) .
$$

In case (I), we have $\lambda=\Theta\left(N^{2}\right)$, thus (3.67) follows. Then using (3.21), we derive

$$
\begin{aligned}
\sum_{k=1}^{N} \operatorname{Var}\left(X_{k, \lambda}\right) & =\sum_{k=1}^{N} \frac{\sqrt{\beta}}{2(k F+\lambda)^{3 / 2}}(1+o(1)) \\
& =\frac{N}{\lambda^{3 / 2}} \frac{\sqrt{\beta}}{\frac{N F}{\lambda}+1+\sqrt{\frac{N F}{\lambda}+1}}(1+o(1))
\end{aligned}
$$

as $\lambda \rightarrow \infty$, and the statement follows by Lemma 3.5.

Similarly, one treats the remaining cases, taking into account Lemma 3.5 and also Remark 3.6.

3.4. Central limit theorem (for the weak force case). Consider

$$
S_{N, \lambda}=\sum_{k=1}^{N} X_{k, \lambda} .
$$

From now on, we assume that $\lambda=\lambda(N, F)$ is chosen so that $\mathbb{E} S_{N, \lambda}=1$ (see Lemma 3.5). We shall also use notation

$$
X_{k, \lambda}=X_{\lambda_{k}},
$$

where as above $\lambda_{k}=k F+\lambda(N, F)$.

Define a random variable

$$
Z_{N}=\frac{S_{N, \lambda}-1}{\sqrt{\operatorname{Var}\left(S_{N, \lambda}\right)}}=\frac{1}{\sigma_{N}} \sum_{k=1}^{N} \xi_{k},
$$

where

$$
\xi_{k}=X_{k, \lambda}-m_{k, \lambda},
$$

and

$$
m_{k, \lambda}=\mathbb{E} X_{k, \lambda}, \quad \sigma_{N}=\sqrt{\operatorname{Var}\left(S_{N, \lambda}\right)}=\sqrt{\sum_{k=1}^{N} \operatorname{Var}\left(X_{k, \lambda}\right)} .
$$

For any random variable $X$ denote $\phi_{X}$ and $f_{X}$ the characteristic function and the density, correspondingly.

Following the method of [17], we shall prove that in the subcritical case [parts (a) and (b) of Theorem 2.2] density $f_{Z_{N}}$ converges to the Normal density. The difference is that here we are dealing with nonidentically distributed random variables. 
LEMMA 3.9 (Central limit theorem). Assume Weak force:

$$
\lim _{N \rightarrow \infty} F / N=F_{0}<4 \beta,
$$

and let $\lambda=\lambda(N, F)$ satisfy (3.18). Then

$$
\left|f_{Z_{N}}(x)-\frac{1}{\sqrt{2 \pi}} e^{-x^{2} / 2}\right| \leq O\left(N^{-1 / 2}\right) .
$$

PROOF. First, we observe that by the assumptions of Lemma 3.9 the value $\lambda=\lambda(N, F)$ is given by the statement (a) weak force of Lemma 3.5, which is

$$
\lambda=\lambda(N, F)=\beta\left(1-\frac{F_{0}}{4 \beta}\right)^{2} N^{2}+O(N) .
$$

Consider now the uniform in $x$ bound obtained with the Fourier inverse formula for the densities

$$
\left|f_{Z_{N}}(x)-\frac{1}{\sqrt{2 \pi}} e^{-x^{2} / 2}\right| \leq \frac{1}{\sqrt{2 \pi}} \int_{-\infty}^{\infty}\left|\phi_{Z_{N}}(t)-e^{-t^{2} / 2}\right| d t .
$$

We shall use the following result of Petrov [18].

LEMMA ([18], page 109). Let $\xi_{1}, \ldots, \xi_{N}$ be independent random variables with $\mathbb{E} \xi_{k}=0$ and $\mathbb{E}\left|\xi_{k}\right|^{3}<\infty$. Define

$$
L_{N}:=\frac{\sum_{k=1}^{N} \mathbb{E}\left|\xi_{k}\right|^{3}}{\sigma_{N}^{3}}
$$

Then for all $|t| \leq \frac{1}{4 L_{N}}$

$$
\left|\phi_{Z_{N}}(t)-e^{-t^{2} / 2}\right| \leq 16 L_{N}\left|t^{3}\right| e^{-t^{2} / 3}
$$

To make use of the bound (3.76), we have to show that $L_{N}$ is decreasing towards zero as $N \rightarrow \infty$.

PROPOSITION 3.10. Under assumptions of Lemma 3.9 (weak force), one has

$$
L_{N}:=\frac{\sum_{k=1}^{N} \mathbb{E}\left|\xi_{k}\right|^{3}}{\sigma_{N}^{3}}=O\left(\frac{1}{\sqrt{N}}\right)
$$

Proof. Consider first for any fixed $k$

$$
\mathbb{E}\left|\xi_{k}\right|^{3}=\mathbb{E}\left|X_{k, \lambda}-m_{k, \lambda}\right|^{3}=\frac{\int_{0}^{1}\left|x-m_{k, \lambda}\right|^{3} e^{-\lambda_{k} x-\frac{\beta}{x}} d x}{\int_{0}^{1} e^{-\lambda_{k} x-\frac{\beta}{x}} d x},
$$


where $\lambda_{k}=\lambda+k F$, and by (3.73) we have here $\lambda_{k} \rightarrow \infty$ as $N \rightarrow \infty$ for any $k$. Recall that by Proposition 3.3,

$$
m_{k, \lambda}=\sqrt{\frac{\beta}{\lambda_{k}}}+O\left(\frac{\beta}{\lambda_{k}}\right)
$$

when $\lambda_{k} \rightarrow \infty$. Let us write here $m_{k, \lambda}=m\left(\lambda_{k}\right)$, and consider

$$
J(\lambda):=\frac{\int_{0}^{1}|x-m(\lambda)|^{3} e^{-\lambda x-\frac{\beta}{x}} d x}{\int_{0}^{1} e^{-\lambda x-\frac{\beta}{x}} d x}
$$

for large $\lambda$. Let us define a function

$$
s(x)=\lambda x+\frac{\beta}{x}, \quad x>0 .
$$

Denote $x_{0}$ the argument of the minimal value of $s(x)$ for $x>0$, which is

$$
x_{0}=\sqrt{\frac{\beta}{\lambda}}
$$

where

$$
s\left(x_{0}\right)=2 \sqrt{\beta \lambda}, \quad s^{\prime}\left(x_{0}\right)=0 \quad \text { and } \quad s^{\prime \prime}\left(x_{0}\right)=\frac{2 \beta}{x_{0}^{3}}=2 \frac{\lambda^{3 / 2}}{\sqrt{\beta}} .
$$

Notice that $x_{0}<1$ since $\lambda \rightarrow \infty$ for all large $N$.

It is straightforward to compute that for any $\varepsilon>-\sqrt{\lambda} x_{0}$ and for all large $\lambda$

$$
s\left(x_{0}+\frac{\varepsilon}{\sqrt{\lambda}}\right) \geq s\left(x_{0}\right)+\frac{\sqrt{\lambda} \varepsilon^{2}}{\sqrt{\beta}+|\varepsilon|} .
$$

Since $s^{\prime}(x)<0$ if $x<x_{0}$ and $s^{\prime}(x)>0$ if $x>x_{0}$, the bounds (3.84) and (3.79) imply for any $0 \leq \alpha \leq 3$ and $0<\varepsilon<x_{0} \sqrt{\lambda}=\sqrt{\beta}$

$$
\begin{aligned}
I(\alpha):= & \int_{0}^{1}|x-m(\lambda)|^{\alpha} e^{-s(x)} d x \\
= & \int_{x_{0}-\frac{\varepsilon}{\sqrt{\lambda}}}^{x_{0}+\frac{\varepsilon}{\sqrt{\lambda}}}\left|x-x_{0}\right|^{\alpha} e^{-s(x)} d x \\
& +\alpha O\left(\left(\frac{\varepsilon}{\sqrt{\lambda}}\right)^{\alpha-1} \frac{1}{\lambda}\right) \int_{0}^{1} e^{-s(x)} d x+e^{-s\left(x_{0}\right)} O\left(e^{-\frac{\sqrt{\lambda} \varepsilon^{2}}{2(\sqrt{\beta}+|\varepsilon|)}}\right) .
\end{aligned}
$$


Next, for any $\varepsilon=o(1)$ we derive using formulas (3.83) and (3.82):

$$
\begin{aligned}
& \int_{x_{0}-\frac{\varepsilon}{\sqrt{\lambda}}}^{x_{0}+\frac{\varepsilon}{\sqrt{\lambda}}}\left|x-x_{0}\right|^{\alpha} e^{-s(x)} d x \\
&=e^{-s\left(x_{0}\right)} \int_{x_{0}-\frac{\varepsilon}{\sqrt{\lambda}}}^{x_{0}+\frac{\varepsilon}{\sqrt{\lambda}}}\left|x-x_{0}\right|^{\alpha} e^{-\frac{1}{2} s^{\prime \prime}\left(x_{0}\right)\left(x-x_{0}\right)^{2}\left(1+O\left(\frac{x-x_{0}}{x_{0}}\right)\right)} d x \\
&= e^{-s\left(x_{0}\right)}(1+O(\varepsilon))\left(\frac{1}{\sqrt{s^{\prime \prime}\left(x_{0}\right)}}\right)^{\alpha+1} \\
& \times \int_{-\frac{\varepsilon}{\sqrt{\lambda}} \sqrt{s^{\prime \prime}\left(x_{0}\right)(1+O(\varepsilon))}}^{\frac{\varepsilon}{\sqrt{\lambda}} \sqrt{s^{\prime \prime}\left(x_{0}\right)(1+O(\varepsilon))}}|x|^{\alpha} e^{-\frac{1}{2} x^{2}} d x \\
&= e^{-s\left(x_{0}\right)}(1+O(\varepsilon))\left(\frac{1}{\sqrt{s^{\prime \prime}\left(x_{0}\right)}}\right)^{\alpha+1}\left(1+O\left(e^{-\frac{\varepsilon^{2} s^{\prime \prime}\left(x_{0}\right)}{4 \lambda}}\right)\right) \\
& \times \int_{-\infty}^{\infty}|x|^{\alpha} e^{-\frac{1}{2} x^{2}} d x .
\end{aligned}
$$

We can choose now

$$
\varepsilon=\frac{\log \lambda}{\lambda^{1 / 4}}=O\left(\frac{\log N}{\sqrt{N}}\right)
$$

[where the last equality is by (3.73)], so that (3.86) combined with (3.85) gives us

$$
\begin{aligned}
I(\alpha)= & e^{-s\left(x_{0}\right)}(1+O(\varepsilon))\left(\frac{1}{\sqrt{s^{\prime \prime}\left(x_{0}\right)}}\right)^{\alpha+1} \int_{-\infty}^{\infty}|x|^{\alpha} e^{-\frac{1}{2} x^{2}} d x \\
& +\alpha O\left(\frac{(\log \lambda)^{\alpha-1}}{\lambda^{1+\frac{\alpha-1}{2}+\frac{\alpha-1}{4}}}\right) I(0)+e^{-s\left(x_{0}\right)} O\left(e^{-\frac{\sqrt{\lambda} \varepsilon^{2}}{2(\sqrt{\beta}+|\varepsilon|)}}\right) .
\end{aligned}
$$

Making use of the last formula with $\alpha=3$ and $\alpha=0$ in (3.80), and taking into account (3.83) we derive for all $\lambda$

$$
\begin{aligned}
J(\lambda) & =\frac{I(3)}{I(0)}=c(1+o(1))\left(\frac{1}{\sqrt{s^{\prime \prime}\left(x_{0}\right)}}\right)^{3}+O\left(\frac{(\log \lambda)^{2}}{\lambda^{2+\frac{1}{2}}}\right) \\
& =c(1+o(1))\left(\frac{\sqrt{\beta}}{2 \lambda^{3 / 2}}\right)^{3 / 2},
\end{aligned}
$$

where

$$
c=\frac{1}{\sqrt{2 \pi}} \int_{-\infty}^{\infty}|x|^{3} e^{-\frac{1}{2} x^{2}} d x .
$$

This together with (3.78) and (3.80) immediately imply

$$
\mathbb{E}\left|\xi_{k}\right|^{3} \leq C\left(\frac{1}{\lambda_{k}}\right)^{9 / 4},
$$

where $C$ is some positive constant. 
Recall that $\lambda_{k}=k F+\lambda$, where $\lambda \geq b N^{2}$ for some positive $b$ [see (3.73)]. Hence, bound (3.90) yields

$$
\sum_{k=1}^{N} \mathbb{E}\left|\xi_{k}\right|^{3} \leq O\left(N^{-7 / 2}\right) .
$$

Finally, we note that by Corollary 3.8 (I) we have $\sigma_{N}^{2} \geq c N^{-2}$ for some positive $c$, which implies $\sigma_{N}^{3} \geq c^{3 / 2} N^{-3}$. The latter bound together with (3.91) yield the statement of the proposition.

Consider again the inequality (3.74). To apply the bound (3.76), we split the integral in (3.74) into three parts (this is a standard procedure; see, e.g., [10]):

$$
\begin{aligned}
\mid f_{Z_{N}}(x) & -\frac{1}{\sqrt{2 \pi}} e^{-x^{2} / 2} \mid \\
\leq & \frac{1}{\sqrt{2 \pi}} \int_{|t| \leq \frac{1}{4 L_{N}}}\left|\phi_{Z_{N}}(t)-e^{-t^{2} / 2}\right| d t \\
& +\frac{1}{\sqrt{2 \pi}} \int_{|t|>\frac{1}{4 L_{N}}}\left|\phi_{Z_{N}}(t)\right| d t+\frac{1}{\sqrt{2 \pi}} \int_{|t|>\frac{1}{4 L_{N}}} e^{-t^{2} / 2} d t .
\end{aligned}
$$

Bounds (3.76) and (3.77) allow us to derive from here

$$
\begin{aligned}
& \left|f_{Z_{N}}(x)-\frac{1}{\sqrt{2 \pi}} e^{-x^{2} / 2}\right| \\
& \leq O\left(L_{N}\right)+\frac{1}{\sqrt{2 \pi}} \int_{|t|>\frac{1}{4 L_{N}}}\left|\phi_{Z_{N}}(t)\right| d t+O\left(e^{-1 / L_{N}}\right) \\
& \quad=O\left(N^{-1 / 2}\right)+\frac{1}{\sqrt{2 \pi}} \int_{|t|>\frac{1}{4 L_{N}}}\left|\phi_{Z_{N}}(t)\right| d t
\end{aligned}
$$

Consider the remaining integral on the right in (3.92). Observe that by the definition (3.71),

$$
\left|\phi_{Z_{N}}(t)\right|=\left|\prod_{k=1}^{N} \phi_{\xi_{k}}\left(\frac{t}{\sqrt{\sigma_{N}^{2}}}\right)\right|=\prod_{k=1}^{N}\left|\phi_{X_{k, \lambda}}\left(\frac{t}{\sqrt{\sigma_{N}^{2}}}\right)\right| .
$$

We shall derive now how fast $\left|\phi_{X_{k, \lambda}}(t)\right|$ decays in $|t|$, as well as we shall get a uniform bound

$$
\sup _{|t|>\frac{1}{4 L_{N}}}\left|\phi_{X_{k, \lambda}}\left(\frac{t}{\sqrt{\sigma_{N}^{2}}}\right)\right| \leq \gamma<1,
$$

for all $k \leq N$ and all large $N$, where $\gamma$ is some constant. 
Consider

$$
\phi_{X_{k, \lambda}}(t)=\int_{0}^{1} e^{i t x} f_{X_{k, \lambda}}(x) d x
$$

where by the definition (3.15)

$$
f_{X_{k, \lambda}}(u)=f_{k, \lambda}(u)=\frac{e^{-\frac{\beta}{u}-(\lambda+F k) u}}{\int_{0}^{1} f_{k, \lambda}(x) d x}, \quad u \in[0,1],
$$

is the density of $X_{k, \lambda}$. Using again notation

$$
\lambda_{k}=\lambda+k F,
$$

we shall write here

$$
f_{X_{k, \lambda}}(u)=f_{\lambda_{k}}(u)
$$

Formula (3.96) gives us

$$
f_{\lambda_{k}}^{\prime}(u)=f_{\lambda_{k}}(u)\left(\frac{\beta}{u^{2}}-\lambda_{k}\right),
$$

which allows us to derive (integrating by parts) from (3.95)

$$
\begin{aligned}
\left|\phi_{X_{k, \lambda}}(t)\right| & \leq \frac{1}{|t|}\left(f_{\lambda_{k}}(1)+\int_{0}^{1}\left|f_{\lambda_{k}}^{\prime}(u)\right| d u\right) \\
& =\frac{1}{|t|}\left(f_{\lambda_{k}}(1)+\int_{0}^{\sqrt{\frac{\lambda_{k}}{\beta}}} f_{\lambda_{k}}^{\prime}(u) d x-\int_{\sqrt{\frac{\lambda_{k}}{\beta}}}^{1} f_{\lambda_{k}}^{\prime}(u) d x\right) \\
& =\frac{2}{|t|} f\left(\sqrt{\frac{\lambda_{k}}{\beta}}\right)=\frac{2}{|t|} \frac{e^{-2 \sqrt{\beta \lambda_{k}}}}{c\left(\lambda_{k}\right)},
\end{aligned}
$$

where

$$
c(\lambda):=\int_{0}^{1} e^{-\frac{\beta}{u}-\lambda u} d x .
$$

This together with (3.28) gives us

$$
\left|\phi_{X_{k, \lambda}}(t)\right| \leq \beta^{-1 / 4} \sqrt{\frac{1}{\pi}} \frac{\lambda_{k}^{3 / 4}}{|t|}\left(1+O\left(\lambda_{k}^{-1 / 2}\right)\right)
$$

as $\lambda_{k} \rightarrow \infty$. Recall that in (3.99) $\lambda_{k}=\lambda+k F$ where $F<4 \beta N$ and $\lambda$ is given by (3.73). Hence, there are positive constants $a<A$ such that

$$
a N^{2}<\lambda_{k}=\lambda+k F<A N^{2}
$$

uniformly in $1 \leq k \leq N$. Therefore, (3.100) yields

$$
\left|\phi_{X_{k, \lambda}}(t)\right|<A_{1} \frac{N^{3 / 2}}{|t|}
$$

for some constant $A_{1}>0$ uniformly in $1 \leq k \leq N$. 
To prove (3.94), observe that $L_{N}=O\left(N^{-1 / 2}\right)$ by Proposition 3.10, while $\sigma_{N}^{2}=$ $O\left(N^{-2}\right)$ by Corollary 3.8 (I) Weak force. Therefore,

$$
\frac{1}{4 L_{N} \sqrt{\sigma_{N}^{2}}} \geq B N^{3 / 2}
$$

for some $B>0$, which yields

$$
\sup _{|t|>\frac{1}{4 L_{N}}}\left|\phi_{X_{k, \lambda}}\left(\frac{t}{\sqrt{\sigma_{N}^{2}}}\right)\right|=\sup _{|s|>\frac{1}{4 L_{N} \sqrt{\sigma_{N}^{2}}}}\left|\phi_{X_{k, \lambda}}(s)\right| \leq \sup _{|s|>B N^{3 / 2}}\left|\phi_{X_{k, \lambda}}(s)\right| .
$$

Consider the last supremum. Bound (3.101) immediately implies that for all positive $d$

$$
\sup _{|s|>d N^{3 / 2}}\left|\phi_{X_{k, \lambda}}(s)\right|<\frac{A_{1}}{d} .
$$

Next, for any constant $d>B$ we shall find an upper bound for

$$
\sup _{B N^{3 / 2}<|s|<d N^{3 / 2}}\left|\phi_{X_{k, \lambda}}(s)\right|=\sup _{B<|b|<d}\left|\phi_{X_{k, \lambda}}\left(b N^{3 / 2}\right)\right| .
$$

Recall again that

$$
\phi_{X_{k, \lambda}}\left(b N^{3 / 2}\right)=\frac{1}{c\left(\lambda_{k}\right)} \int_{0}^{1} e^{-\frac{\beta}{u}-\left(\lambda_{k}-i b N^{3 / 2}\right) u} d u .
$$

Notice that when $a N^{2}<\lambda_{k}<A N^{2}$ we have $\left|\arg \left(\lambda_{k}-i b N^{3 / 2}\right)\right|<\pi / 8$ for any real-valued constant $b$ when $N$ is large. Therefore, by Remark 3.4 we have here as in (3.28)

$$
\begin{aligned}
& \left|\int_{0}^{1} e^{-\frac{\beta}{u}-\left(\lambda_{k}-i b N^{3 / 2}\right) u} d u\right| \\
& \quad=\left|\int_{0}^{\infty} e^{-\frac{\beta}{u}-\left(\lambda_{k}-i b N^{3 / 2}\right) u} d u\right|+O\left(e^{-\lambda_{k}}\right) \\
& \quad=\left|\sqrt{\pi} \beta^{1 / 4} \frac{e^{-2 \sqrt{\beta\left(\lambda_{k}-i b N^{3 / 2}\right)}}}{\left(\lambda_{k}-i b N^{3 / 2}\right)^{3 / 4}}\right|(1+o(1))+O\left(e^{-\lambda_{k}}\right) \\
& \quad=\sqrt{\pi} \frac{\beta^{1 / 4}}{\lambda_{k}^{3 / 4}} e^{-2 \sqrt{\beta \lambda_{k}} \operatorname{Re}\left(\sqrt{1-i b \frac{N^{3 / 2}}{\lambda_{k}}}\right)}(1+o(1))+O\left(e^{-\lambda_{k}}\right) .
\end{aligned}
$$

Making use of

$$
\operatorname{Re}(\sqrt{1-i \varepsilon})=1+\frac{\varepsilon^{2}}{2}+O\left(\varepsilon^{4}\right), \quad|\varepsilon| \rightarrow 0,
$$


we derive from (3.106)

$$
\left|\int_{0}^{1} e^{-\frac{\beta}{u}-\left(\lambda_{k}-i b N^{3 / 2}\right) u} d u\right|=\sqrt{\pi} \frac{\beta^{1 / 4}}{\lambda_{k}^{3 / 4}} e^{-2 \sqrt{\beta \lambda_{k}}\left(1+b^{2} \frac{N^{3}}{2 \lambda_{k}^{2}}\right)}(1+o(1))+O\left(e^{-\lambda_{k}}\right),
$$

where $o(1)$ is uniform in $k \leq N$. Substituting this and (3.28) into (3.105), we get

$$
\left|\phi_{X_{k, \lambda}}\left(b N^{3 / 2}\right)\right|=e^{-\sqrt{\beta \lambda_{k}} b^{2} \frac{N^{3}}{\lambda_{k}^{2}}}(1+o(1))+O\left(e^{-\lambda_{k} / 2}\right) .
$$

This together with the assumption $a N^{2} \leq \lambda_{k} \leq A N^{2}$ implies for any fixed constant $d$

$$
\sup _{B<|b|<d}\left|\phi_{X_{k, \lambda}}\left(b N^{3 / 2}\right)\right| \leq e^{-\sqrt{\beta} \frac{B^{2}}{A^{3 / 2}}}(1+o(1))+O\left(e^{-N}\right) \leq \gamma<1
$$

for some positive $\gamma$ and all large $N$ uniformly in $k$.

Finally, choosing constant $d$ large enough and combining bounds (3.108) and (3.103) together with (3.102) we obtain claim (3.94).

Consider now the last integral in (3.92) taking into account (3.93):

$$
\begin{aligned}
\int_{|t|>\frac{1}{4 L_{N}}}\left|\phi_{Z_{N}}(t)\right| d t & =\int_{|t|>\frac{1}{4 L_{N}}} \prod_{k=1}^{N}\left|\phi_{X_{k, \lambda}}\left(\frac{t}{\sqrt{\sigma_{N}^{2}}}\right)\right| d t \\
\leq & \left.\max _{3 \leq k \leq N} \sup _{|t|>\frac{1}{4 L_{N}}}\left|\phi_{X_{k, \lambda}}\left(\frac{t}{\sqrt{\sigma_{N}^{2}}}\right)\right|\right)^{N-2} \\
& \times \int_{|t|>\frac{1}{4 L_{N}}} \prod_{k=1}^{2}\left|\phi_{X_{k, \lambda}}\left(\frac{t}{\sqrt{\sigma_{N}^{2}}}\right)\right| d t
\end{aligned}
$$

Making use of the uniform bounds (3.94) and (3.101), we derive from here

$$
\int_{|t|>\frac{1}{4 L_{N}}}\left|\phi_{Z_{N}}(t)\right| d t \leq \gamma^{N-2} \int_{|t|>\frac{1}{4 L_{N}}} A_{1}^{2} \frac{N^{3}}{|t|^{2}} d t \leq \gamma^{N} O\left(N^{3}\right) .
$$

Substituting the last bound into (3.92) we get the statement of Lemma 3.9.

COROLLARY 3.11. Under assumptions of Lemma 3.9 (weak force) one has

$$
\begin{aligned}
f_{S_{N, \lambda}}(x) & =\frac{1}{\sqrt{2 \pi \sigma_{N}^{2}}} e^{-\frac{(x-1)^{2}}{2 \sigma_{N}^{2}}}+O\left(N^{-1 / 2}\right) \\
& =\frac{1}{\sqrt{2 \pi \sigma_{N}^{2}}}\left(e^{-\frac{(x-1)^{2}}{2 \sigma_{N}^{2}}}+O\left(N^{-3 / 2}\right)\right),
\end{aligned}
$$


as well as

$$
f_{S_{N, \hat{k}, \lambda}}(x)=\frac{1}{\sqrt{2 \pi \sigma_{N}^{2}}}\left(e^{-\frac{\left(x-1+\mathbb{E} X_{k, \lambda}\right)^{2}}{2 \sigma_{N}^{2}}}+O\left(N^{-3 / 2}\right)\right)
$$

uniformly in $1 \leq k \leq N$ and $x \in \mathbb{R}$.

PROOF. Formula (3.110) follows immediately by Lemma 3.9 and formula (3.71), while (3.111) follows by the same argument and Corollary 3.8 [(I) Weak force].

REMARK 3.12. Observe that in the case of weak external force (i.e., $F / N<$ $4 \beta$ ) result (3.110) together with (3.28) yield the exact asymptotic for the partition function (3.19).

Now we turn to the proof of the statements of Theorem 2.2.

3.5. Proof of Theorem 2.2 in the subcritical phases $(a)$ and $(b)$. Assume that $F(N) / N=F_{0}<4 \beta$. In this case, we have by Lemma 3.5 [(a) Weak force],

$$
\lambda_{k}=k F+\lambda=\beta N^{2}+\left(k-\frac{N}{2}\right) F+\beta\left(\frac{F}{4 \beta}\right)^{2}+O(N) .
$$

Applying (3.17) and using the result of the last Corollary 3.11, we get

$$
\begin{aligned}
\mathbb{E}\left\{X_{k} \mid \sum_{i=1}^{N} X_{i}=1\right\} & =\int_{0}^{1} x \frac{f_{k, \lambda}(x) f_{S_{N, \hat{k}, \lambda}}(1-x)}{f_{S_{N, \lambda}}(1)} d x \\
& =\int_{0}^{1} x f_{k, \lambda}(x)\left(e^{-\frac{\left(x-\mathbb{E} X_{k, \lambda}\right)^{2}}{2 \sigma_{N}^{2}}}+O\left(N^{-3 / 2}\right)\right) d x \\
& =\int_{0}^{1} x f_{k, \lambda}(x) e^{-\frac{\left(x-\mathbb{E} X_{k, \lambda}\right)^{2}}{2 \sigma_{N}^{2}}} d x+O\left(N^{-3 / 2}\right) \mathbb{E} X_{k, \lambda} .
\end{aligned}
$$

Here, by Proposition 3.3,

$$
\mathbb{E} X_{k, \lambda}=\sqrt{\frac{\beta}{\lambda_{k}}}+O\left(1 / \lambda_{k}\right)
$$

and

$$
\sigma_{N}^{2}=C N^{-2}(1+o(1))
$$

for some positive $C$ by Corollary 3.8 (I) (Weak force). 
Consider now the last integral in (3.113)

$$
\mathcal{I}\left(\lambda_{k}\right)=\int_{0}^{1} x f_{k, \lambda}(x) e^{-\frac{\left(x-\mathbb{E} X_{k, \lambda}\right)^{2}}{2 \sigma_{N}^{2}}} d x
$$

$$
=\frac{1}{I_{1}\left(\lambda_{k}, \beta\right)+O\left(e^{-\lambda / 2}\right)} \int_{0}^{1} x e^{-\lambda_{k} x-\frac{\beta}{x}} e^{-\frac{\left(x-\sqrt{\frac{\beta}{\lambda_{k}}}+O\left(1 / \lambda_{k}\right)\right)^{2}}{2 \sigma_{N}^{2}}} d x,
$$

where we used notation (3.23). For all $\lambda>c N^{2}$ and any positive constant $a$, we have

$$
\mathcal{I}(\lambda)=\frac{1}{I_{1}(\lambda, \beta)+O\left(e^{-\lambda / 2}\right)}
$$

$$
\times\left(\int_{0}^{a / \sqrt{\lambda}} x e^{-\lambda x-\frac{\beta}{x}} e^{-\frac{\left(x-\sqrt{\frac{\beta}{\lambda}}+O(1 / \lambda)\right)^{2}}{2 \sigma_{N}^{2}}} d x+O\left(e^{-a \sqrt{\lambda} / 2}\right)\right) .
$$

Recall that by (3.25) and (3.26),

$$
I_{1}(\lambda, \beta)=\sqrt{\frac{\beta}{\lambda}} \sqrt{\pi}(\lambda \beta)^{-1 / 4} e^{-2 \sqrt{\beta \lambda}}(1+O(1 / \sqrt{\lambda})) .
$$

Hence, choosing $a=8 \sqrt{\beta}$ we get

$$
\frac{O\left(e^{-a \sqrt{\lambda} / 2}\right)}{I_{1}(\lambda, \beta)+O\left(e^{-\lambda / 2}\right)}=O\left(e^{-\sqrt{\beta \lambda}}\right) .
$$

This together with (3.117) and observation that for all $x \in[0, a / \sqrt{\lambda}]$,

$$
e^{-\frac{\left(x-\sqrt{\frac{\beta}{\lambda}}+O(1 / \lambda)\right)^{2}}{2 \sigma_{N}^{2}}}=e^{-\frac{\left(x-\sqrt{\frac{\beta}{\lambda}}\right)^{2}}{2 \sigma_{N}^{2}}} e^{\frac{O(1 / \lambda) O(1 / \sqrt{\lambda})}{2 \sigma_{N}^{2}}}=e^{-\frac{\left(x-\sqrt{\frac{\beta}{\lambda}}\right)^{2}}{2 \sigma_{N}^{2}}} e^{O(1 / N)}
$$

gives us

$$
\mathcal{I}(\lambda)=\frac{1}{I_{1}(\lambda, \beta)+O\left(e^{-\lambda / 2}\right)}
$$

$$
\times \int_{0}^{a / \sqrt{\lambda}} x e^{-\lambda x-\frac{\beta}{x}} e^{-\frac{\left(x-\sqrt{\frac{\beta}{\lambda}}\right)^{2}}{2 \sigma_{N}^{2}}} d x\left(1+O\left(\frac{1}{N}\right)\right)+O\left(e^{-\sqrt{\beta \lambda}}\right) .
$$

Notice that in notation (3.82)

$$
a / \sqrt{\lambda}=8 \sqrt{\frac{\beta}{\lambda}}=8 x_{0}
$$

Then using (3.84) and

$$
\varepsilon=\frac{\log \lambda}{\lambda^{1 / 4}}
$$


[defined as in (3.87)], we obtain, taking into account (3.112),

$$
\begin{aligned}
& \int_{0}^{a / \sqrt{\lambda}} x e^{-\lambda x-\frac{\beta}{x}} e^{-\frac{\left(x-\sqrt{\frac{\beta}{\lambda}}\right)^{2}}{2 \sigma_{N}^{2}}} d x \\
&=\int_{\left|x-x_{0}\right|<\varepsilon / \sqrt{\lambda}} x e^{-\lambda x-\frac{\beta}{x}} e^{-\frac{\left(x-\sqrt{\frac{\beta}{\lambda}}\right)^{2}}{2 \sigma_{N}^{2}}} d x+O\left(e^{\left.-s\left(x_{0}\right)-\frac{\sqrt{\lambda} \varepsilon^{2}}{2(\sqrt{\beta+a})}\right)}\right. \\
&=\int_{\left|x-x_{0}\right|<\varepsilon / \sqrt{\lambda}} x e^{-\lambda x-\frac{\beta}{x}} d x\left(1+O\left(\frac{\varepsilon^{2}}{\lambda \sigma_{N}^{2}}\right)\right)+O\left(e^{-s\left(x_{0}\right)-\frac{\sqrt{\lambda} \varepsilon^{2}}{2(\sqrt{\beta+a})}}\right) \\
&=\left(x_{0}+O\left(\frac{\varepsilon}{\sqrt{\lambda}}\right)\right)\left(1+O\left(\frac{\varepsilon^{2}}{\lambda \sigma_{N}^{2}}\right)\right) \int_{\left|x-x_{0}\right|<\varepsilon / \sqrt{\lambda}} e^{-\lambda x-\frac{\beta}{x}} d x \\
&+O\left(e^{-s\left(x_{0}\right)-\frac{\sqrt{\lambda} \varepsilon^{2}}{2(\sqrt{\beta+a})}}\right),
\end{aligned}
$$

which by (3.86) and (3.115) yields

$$
\begin{aligned}
& \int_{0}^{a / \sqrt{\lambda}} x e^{-\lambda x-\frac{\beta}{x}} e^{-\frac{\left(x-\sqrt{\frac{\beta}{\lambda}}\right)^{2}}{2 \sigma_{N}^{2}}} d x \\
& \quad=x_{0}(1+O(\varepsilon)) e^{-s\left(x_{0}\right)}\left(s^{\prime \prime}\left(x_{0}\right)\right)^{-1 / 2} \sqrt{2 \pi}+O\left(e^{-s\left(x_{0}\right)-\frac{\sqrt{\lambda}^{2}}{2(\sqrt{\beta+a})}}\right) .
\end{aligned}
$$

Substituting this and formula (3.118) into (3.119), we derive that

$$
\mathcal{I}(\lambda)=\sqrt{\frac{\beta}{\lambda}}\left(1+O\left(\frac{\log N}{\sqrt{N}}\right)\right)+O\left(e^{-\sqrt{\beta \lambda}}\right)=\sqrt{\frac{\beta}{\lambda}}\left(1+O\left(\frac{(\log N)^{2}}{\sqrt{N}}\right)\right)
$$

Recall that by (3.113) and (3.116)

$$
\mathbb{E}\left\{X_{k} \mid \sum_{i=1}^{N} X_{i}=1\right\}=\mathcal{I}\left(\lambda_{k}\right)+O\left(N^{-3 / 2}\right) \mathbb{E} X_{k, \lambda},
$$

which together with (3.121) and (3.114) yields

$$
\mathbb{E}\left\{X_{k} \mid \sum_{i=1}^{N} X_{i}=1\right\}=\sqrt{\frac{\beta}{\lambda_{k}}}\left(1+O\left(\frac{\log N}{\sqrt{N}}\right)\right) .
$$

Substituting here (3.112), we derive

$$
\mathbb{E}\left\{X_{k} \mid \sum_{i=1}^{N} X_{i}=1\right\}=\sqrt{\frac{1}{N^{2}+\left(k-\frac{N}{2}\right) \frac{F}{\beta}+\frac{F^{2}}{16 \beta^{2}}}}\left(1+O\left(\frac{\log N}{\sqrt{N}}\right)\right),
$$


which due to the identity (3.14) is equivalent to

$$
\begin{aligned}
& \mathbb{E}\left\{Y_{N-k+1}-Y_{N-k}\right\} \\
& \quad=\frac{1}{N} \sqrt{\frac{1}{1+\left(\frac{k}{N}-\frac{1}{2}\right) \frac{F}{N \beta}+\frac{F^{2}}{16 N^{2} \beta^{2}}}}\left(1+O\left(\frac{\log N}{\sqrt{N}}\right)\right) .
\end{aligned}
$$

Finally, to derive the statements of Theorem 2.2 we have to apply the transformation (3.4) to the parameters $\beta$ and $F$ in the last formula. Notice that the ratio $\frac{F}{\beta}$ is invariant under the latter transformation, and so does the principal term on the right in (3.123) [which yields formula (2.12)]. Hence, for all $t>0$ the statement (2.7) follows by (3.123) when $F=o(N)$, while the statement (2.10) follows by (3.123) when $F=F_{0} N$ for some $0<F_{0}<4 \beta$.

In a fashion similar to (3.113), consider

$$
\begin{aligned}
\mathbb{E}\left\{\left(X_{k}-\sqrt{\frac{\beta}{\lambda_{k}}}\right)^{2} \mid \sum_{i=1}^{N} X_{i}=1\right\} \\
=\int_{0}^{1}\left(x-\sqrt{\frac{\beta}{\lambda_{k}}}\right)^{2} \frac{f_{k, \lambda}(x) f_{S_{N, \hat{k}, \lambda}}(1-x)}{f_{S_{N, \lambda}}(1)} d x \\
=\int_{0}^{1}\left(x-\sqrt{\frac{\beta}{\lambda_{k}}}\right)^{2} f_{k, \lambda}(x) e^{-\frac{\left(x-\mathbb{E} X_{k, \lambda}\right)^{2}}{2 \sigma_{N}^{2}}} d x+O\left(N^{-3 / 2}\right) \operatorname{Var}\left(X_{k, \lambda}\right) .
\end{aligned}
$$

From (3.124), following the same strategy as we used deriving (3.121) from (3.113), we get

$$
\mathbb{E}\left\{\left(X_{k}-\sqrt{\frac{\beta}{\lambda_{k}}}\right)^{2} \mid \sum_{i=1}^{N} X_{i}=1\right\}=\operatorname{Var}\left(X_{k, \lambda}\right)\left(1+O\left(\frac{\log N}{\sqrt{N}}\right)\right) .
$$

By the identity (3.14) this gives us

$$
\operatorname{Var}\left(Y_{N-k+1}-Y_{N-k}\right)=\operatorname{Var}\left(X_{k, \lambda}\right)\left(1+O\left(\frac{\log N}{\sqrt{N}}\right)\right),
$$

where by (3.21)

$$
\operatorname{Var}\left(X_{k, \lambda}\right)=\frac{\sqrt{\beta}}{2 \lambda_{k}^{3 / 2}}(1+o(1))
$$

as $\lambda_{k} \rightarrow \infty$. Then taking into account (3.112), we derive

$$
\begin{aligned}
\operatorname{Var} & \left.Y_{N-k+1}-Y_{N-k}\right) \\
& =\frac{\sqrt{\beta}}{2 \lambda_{k}^{3 / 2}}(1+o(1)) \\
& =\frac{1}{2 \beta N^{3}\left(1+\left(\frac{k}{N}-\frac{1}{2}\right) \frac{F}{\beta N}+\beta\left(\frac{F}{4 \beta N}\right)^{2}\right)^{3 / 2}}(1+o(1)) .
\end{aligned}
$$


Finally, after the transformation (3.4) of the parameters in (3.128) we derive for any $t>0$ both statements (2.8) and (2.11) of the Theorem 2.2: (2.8) follows by (3.128) when $F=o(N)$, while (2.11) follows by (3.128) when $F=F_{0} N / t$, and in both cases $\beta$ is replaced by $\beta / t$.

3.6. Proof of Theorem 2.2 in the supercritical phase (d). Let $F / N=F_{0}$ be a constant such that $F_{0}>4 \beta$. Consider first

$$
\mathbb{E}\left\{X_{1} \mid \sum_{i=1}^{N} X_{i}=1\right\}=\int_{0}^{1} x \frac{f_{1, \lambda}(x) f_{\left(S_{N}-X_{1, \lambda}\right), \lambda}(1-x)}{f_{S_{N, \lambda}}(1)} d x,
$$

where by (3.15) and Lemma 3.5 (c) (Strong force)

$$
f_{1, \lambda}(x)=\frac{e^{-\frac{\beta}{x}+\lambda_{0} x+o\left(1 / \sqrt{F_{0} N}\right) x}}{c}
$$

for some finite constant $\lambda_{0}$, and normalizing factor $c$ of order constant. Recall that by (3.61) we have here

$$
\mathbb{E} X_{1, \lambda}=1-\sqrt{\frac{4 \beta}{F_{0}}}+O\left(\frac{1}{\sqrt{F_{0} N}}\right)=: m_{1} .
$$

Let us write

$$
\Sigma_{2}=S_{N}-X_{1, \lambda}=\sum_{k=2}^{N} X_{k, \lambda}
$$

Note that by Lemma 3.5 here $\mathbb{E} \Sigma_{2}=1-m_{1}$, and by the Corollary 3.8 [(III) Strong force] the variance of $\Sigma_{2}$ decays as $F^{-3 / 2}$, that is, as $N^{-3 / 2}$. Therefore, although we cannot apply the central limit theorem, some concentration results still hold. Consider for $\alpha=0,1,2$, and any $\varepsilon<m_{1} / 2$

$$
\begin{aligned}
J(\alpha):= & \int_{0}^{1} x^{\alpha} f_{1, \lambda}(x) f_{\Sigma_{2}}(1-x) d x \\
= & \int_{\left|x-m_{1}\right|>\varepsilon} O(1) f_{\Sigma_{2}}(1-x) d x \\
& +\left(m_{1}+O(\varepsilon)\right)^{\alpha} \int_{\left|x-m_{1}\right| \leq \varepsilon} f_{1, \lambda}(x) f_{\Sigma_{2}}(1-x) d x .
\end{aligned}
$$

Note that here by Corollary 3.8 (I I I) and Chebyshev's inequality

$$
\int_{\left|x-m_{1}\right|>\varepsilon} f_{\Sigma_{2}}(1-x) d x=\mathbb{P}\left\{\left|\Sigma_{2}-\left(1-m_{1}\right)\right|>\varepsilon\right\}=O\left(\frac{1}{F^{3 / 2} \varepsilon^{2}}\right),
$$

while

$$
\int_{\left|x-m_{1}\right| \leq \varepsilon} f_{1, \lambda}(x) f_{\Sigma_{2}}(1-x) d x \geq \min _{\left|x-m_{1}\right| \leq \varepsilon} f_{1, \lambda}(x)\left(1-O\left(\frac{1}{F^{3 / 2} \varepsilon^{2}}\right)\right),
$$


where $\min _{\left|x-m_{1}\right| \leq \varepsilon} f_{1, \lambda}(x)$ is bounded away from zero uniformly in $\varepsilon<m_{1} / 2$ [see (3.131)]. Notice that (3.134) and (3.130) yield, in particular, that for some positive $\delta\left(\lambda_{0}, \beta\right)$,

$$
J(0)=f_{S_{N, \lambda}}(1)>\delta>0 .
$$

Hence,

$$
\begin{aligned}
J(\alpha) & =\left(O\left(\frac{1}{F^{3 / 2} \varepsilon^{2}}\right)+\left(m_{1}+O(\varepsilon)\right)^{\alpha}\right) \int_{0}^{1} f_{1, \lambda}(x) f_{\Sigma_{2}}(1-x) d x \\
& =\left(O\left(\frac{1}{F^{3 / 2} \varepsilon^{2}}\right)+\left(m_{1}+O(\varepsilon)\right)^{\alpha}\right) f_{S_{N, \lambda}}(1) .
\end{aligned}
$$

Making use of (3.136) (with $\alpha=1$ ) in (3.129), we derive

$$
\mathbb{E}\left\{X_{1} \mid \sum_{i=1}^{N} X_{i}=1\right\}=m_{1}+O(\varepsilon)+O\left(\frac{1}{F^{3 / 2} \varepsilon^{2}}\right) .
$$

Choosing $\varepsilon=1 / \sqrt{N}$, we get from here

$$
\mathbb{E}\left\{X_{1} \mid \sum_{i=1}^{N} X_{i}=1\right\}=m_{1}+O\left(\frac{1}{\sqrt{N}}\right)=1-\sqrt{\frac{4 \beta}{F_{0}}}+O\left(\frac{1}{\sqrt{N}}\right),
$$

where the last equality is by (3.131). This, by the identity (3.14), is equivalent to

$$
\mathbb{E}\left(Y_{N}-Y_{N-1}\right)=1-\sqrt{\frac{4 \beta}{F_{0}}}+O\left(\frac{1}{\sqrt{N}}\right),
$$

which after transformation (3.4), that is, $\beta \rightarrow \beta / t, F_{0} \rightarrow F_{0} / t$, confirms (2.15).

In a similar fashion making use of (3.136) with $\alpha=2$ and taking into account (3.138) we get for all positive $\varepsilon<m_{1} / 2$

$$
\begin{aligned}
\operatorname{Var}\left(Y_{N}-Y_{N-1}\right) & =\mathbb{E}\left\{\left(X_{1}-m_{1}\right)^{2} \mid \sum_{i=1}^{N} X_{i}=1\right\}+O\left(\frac{1}{\sqrt{N}}\right) \\
& =O\left(\frac{1}{F^{3 / 2} \varepsilon^{2}}\right)+O(\varepsilon)+O\left(\frac{1}{\sqrt{N}}\right) .
\end{aligned}
$$

Choosing here again $\varepsilon=1 / \sqrt{N}$, we get

$$
\operatorname{Var}\left(Y_{N}-Y_{N-1}\right)=\operatorname{Var}\left(Y_{N-1}\right)=O\left(\frac{1}{\sqrt{N}}\right),
$$

which confirms (2.16).

Next, we consider $\mathbb{E}\left\{X_{k} \mid \sum_{i=1}^{N} X_{i}=1\right\}=\mathbb{E}\left\{X_{k, \lambda} \mid \sum_{i=1}^{N} X_{i, \lambda}=1\right\}$ for $k>1$. Recall that here

$$
\lambda_{k}=(k-1) F-\lambda_{0}+o(1 / \sqrt{F}),
$$


where by the assumption $F=F_{0} N$, and by the Lemma 3.5 [(c) Strong force $\lambda_{0}=$ $\lambda_{0}\left(F_{0}\right)$ is some constant. Since $\lambda_{k} \rightarrow \infty$ for all $k \geq 2$, by Proposition 3.3, we have here

$$
\mathbb{E} X_{k, \lambda}=m_{k}=\sqrt{\frac{\beta}{\lambda_{k}}}+O\left(\frac{1}{(k-1) F}\right) .
$$

The density of $X_{k, \lambda}$ is

$$
f_{k, \lambda}(x)=\frac{e^{-\frac{\beta}{x}-\lambda_{k} x}}{\int_{0}^{1} e^{-\frac{\beta}{x}-\lambda_{k} x} d x}=\frac{e^{-\frac{\beta}{x}-\lambda_{k} x}}{I_{1}\left(\lambda_{k}, \beta\right)+O\left(e^{-\lambda_{k} / 2}\right)},
$$

where we used notation from (3.23). Using (3.25) with (3.26) and also bound (3.84), we derive

$$
\max _{x:\left|x-m_{k}\right|>\varepsilon / \sqrt{\lambda_{k}}} f_{k, \lambda}(x) \leq O\left(e^{-\frac{\sqrt{\lambda_{k}} \varepsilon^{2}}{2(\sqrt{\beta}+\varepsilon)}}\right) .
$$

Let us write here

$$
\Sigma_{2, \hat{k}}=\Sigma_{2}-X_{k, \lambda}
$$

whose density we denote $f_{\Sigma_{2, \hat{k}}}$. In these notations, we have

$$
\begin{aligned}
& \mathbb{E}\left\{X_{k} \mid \sum_{i=1}^{N} X_{i}=1\right\} \\
& \quad=\frac{1}{f_{S_{N, \lambda}}(1)} \int_{0}^{1}\left(\int_{0}^{z} x f_{k, \lambda}(x) f_{1, \lambda}(z-x) d x\right) f_{\Sigma_{2, \hat{k}}}(1-z) d z
\end{aligned}
$$

Consider now for $\alpha=0,1$

$$
g_{\alpha}(z)=\int_{0}^{z} x^{\alpha} f_{k, \lambda}(x) f_{1, \lambda}(z-x) d x .
$$

Using bound (3.142), we derive first for any positive $\varepsilon=o(1)<1$ and $z>m_{k}+$ $\varepsilon / \sqrt{\lambda_{k}}$

$$
\begin{aligned}
g_{1}(z)= & \left(m_{k}+O\left(\frac{\varepsilon}{\sqrt{\lambda_{k}}}\right)\right) \int_{\left|x-m_{k}\right|<\varepsilon / \sqrt{\lambda_{k}}} f_{k, \lambda}(x) f_{1, \lambda}(z-x) d x \\
& +O\left(e^{-\frac{\sqrt{\lambda_{k}} \varepsilon^{2}}{2(\sqrt{\beta}+1)}}\right) \\
= & \left(m_{k}+O\left(\frac{\varepsilon}{\sqrt{\lambda_{k}}}\right)\right)\left(g_{0}(z)-O\left(e^{-\frac{\sqrt{\lambda_{k}} \varepsilon^{2}}{2(\sqrt{\beta}+1)}}\right)\right)+O\left(e^{-\frac{\sqrt{\lambda_{k}} \varepsilon^{2}}{2(\sqrt{\beta}+1)}}\right) \\
= & \left(m_{k}+O\left(\frac{\varepsilon}{\sqrt{\lambda_{k}}}\right)\right) g_{0}(z)+O\left(e^{-\frac{\sqrt{\lambda_{k}} \varepsilon^{2}}{2(\sqrt{\beta}+1)}}\right) .
\end{aligned}
$$


On the other hand, for all $z \leq m_{k}+\varepsilon / \sqrt{\lambda_{k}}$ it holds by (3.130) that

$$
g_{\alpha}(z) \leq z^{\alpha} \max _{0<x<z} f_{1, \lambda}(x)=O\left(z^{\alpha+1}\right),
$$

as $z \rightarrow 0$. With a help of the last bound and (3.144), consider the integral in (3.143):

$$
\begin{aligned}
\int_{0}^{1} g_{1}(z) f_{\Sigma_{2, \hat{k}}}(1-z) d z \\
=\int_{0}^{m_{k}+\varepsilon / \sqrt{\lambda_{k}}} O\left(z^{2}\right) f_{\Sigma_{2, \hat{k}}}(1-z) d z \\
\quad+\int_{m_{k}+\varepsilon / \sqrt{\lambda_{k}}}^{1}\left(O\left(e^{-\frac{\sqrt{\lambda_{k}}}{2(\sqrt{\beta}+1)} \varepsilon^{2}}\right)\right. \\
\left.+\left(m_{k}+O\left(\frac{\varepsilon}{\sqrt{\lambda_{k}}}\right)\right) g_{0}(z)\right) f_{\Sigma_{2, \hat{k}}}(1-z) d z \\
=O\left(m_{k}^{2}\right)+O\left(e^{-\frac{\sqrt{\lambda_{k}}}{2(\sqrt{\beta}+1)} \varepsilon^{2}}\right) \\
+\left(m_{k}+O\left(\frac{\varepsilon}{\sqrt{\lambda_{k}}}\right)\right)\left(\int_{0}^{1} g_{0}(z) f_{\Sigma_{2, \hat{k}}}(1-z) d z-O\left(m_{k}\right)\right) \\
=O\left(m_{k}^{2}\right)+O\left(e^{-\frac{\sqrt{\lambda_{k}}}{2(\sqrt{\beta}+1)} \varepsilon^{2}}\right)+\left(m_{k}+O\left(\frac{\varepsilon}{\sqrt{\lambda_{k}}}\right)\right) f_{S_{N, \lambda}}(1) .
\end{aligned}
$$

Choosing

$$
\varepsilon=\frac{\log N}{\lambda_{k}^{1 / 4}},
$$

allows us to derive from (3.146) and (3.143) [take into account (3.135)] that

$$
\mathbb{E}\left\{X_{k} \mid \sum_{i=1}^{N} X_{i}=1\right\}=m_{k}+O\left(\frac{\log N}{\lambda_{k}^{3 / 4}}\right) .
$$

This by the identity (3.14) [and formula (3.140)] is equivalent to

$$
\begin{aligned}
\mathbb{E}\left(Y_{N+1-k}-Y_{N-k}\right) & =m_{k}+O\left(\frac{\log N}{\lambda_{k}^{3 / 4}}\right) \\
& =\sqrt{\frac{\beta}{(k-1) F-\lambda_{0}}}+O\left(\frac{\log N}{(k N)^{3 / 4}}\right),
\end{aligned}
$$


where $\lambda_{0}$ by Lemma 3.5 (c) satisfies

$$
\frac{\int_{0}^{1} x e^{\lambda_{0} x-\frac{\beta}{x}} d x}{\int_{0}^{1} e^{\lambda_{0} x-\frac{\beta}{x}} d x}=1-\sqrt{\frac{4 \beta}{F_{0}}}
$$

Hence, after transformation (3.4) we get from (3.148)

$$
\mathbb{E}\left(Y_{N+1-k}-Y_{N-k}\right)=\sqrt{\frac{\beta}{(k-1) F-t \lambda_{0}(t)}}+O\left(\frac{\log N}{(k N)^{3 / 4}}\right),
$$

where $\lambda_{0}(t)$ is the solution to (3.149) but with the parameters transformed according to (3.4), that is, $\lambda_{0}(t)$ is the solution to (2.19). This confirms (2.17).

Consider now for $k \geq 2$

$$
\begin{aligned}
\mathbb{E}\left\{\left(X_{k}-m_{k}\right)^{2} \mid \sum_{i=1}^{N} X_{i}=1\right\} \\
=\frac{1}{f_{S_{N, \lambda}}(1)} \int_{0}^{1}\left(\int_{0}^{z}\left(x-m_{k}\right)^{2} f_{k, \lambda}(x) f_{1, \lambda}(z-x) d x\right) \\
\quad \times f_{\Sigma_{2, \hat{k}}}(1-z) d z .
\end{aligned}
$$

Using again the same argument as above in (3.144), first we derive for any positive $\varepsilon=o(1)<1$ and $z>m_{k}+\varepsilon / \sqrt{\lambda_{k}}$

$$
\begin{aligned}
G(z) & :=\int_{0}^{z}\left(x-m_{k}\right)^{2} f_{k, \lambda}(x) f_{1, \lambda}(z-x) d x \\
& =O\left(\frac{\varepsilon^{2}}{\lambda_{k}}\right) \int_{\left|x-m_{k}\right|<\varepsilon / \sqrt{\lambda_{k}}} f_{k, \lambda}(x) f_{1, \lambda}(z-x) d x+O\left(e^{-\frac{\sqrt{\lambda_{k}} \varepsilon^{2}}{2(\sqrt{\beta}+1)}}\right) \\
& =O\left(\frac{\varepsilon^{2}}{\lambda_{k}}\right) g_{0}(z)+O\left(e^{-\frac{\sqrt{\lambda_{k}} \varepsilon^{2}}{2(\sqrt{\beta}+1)}}\right) .
\end{aligned}
$$

On the other hand, for all $z \leq m_{k}+\varepsilon / \sqrt{\lambda_{k}}$ it holds by (3.130)

$$
G(z) \leq O\left(z^{2}\right) \max _{0<x<z} f_{1, \lambda}(x)=O\left(z^{3}\right) \quad \text { as } z \rightarrow 0
$$


Then [similar to (3.146)] we derive using (3.152) in combination with (3.153) and (3.145) that

$$
\begin{aligned}
& \int_{0}^{1} G(z) f_{\Sigma_{2, \hat{k}}}(1-z) d z \\
&=\int_{0}^{m_{k}+\varepsilon / \sqrt{\lambda_{k}}} O\left(z^{3}\right) f_{\Sigma_{2, \hat{k}}}(1-z) d z \\
& \quad+\int_{m_{k}+\varepsilon / \sqrt{\lambda_{k}}}^{1}\left(O\left(e^{-\frac{\sqrt{\lambda_{k}}}{2(\sqrt{\beta}+1)} \varepsilon^{2}}\right)+O\left(\frac{\varepsilon^{2}}{\lambda_{k}}\right) g_{0}(z)\right) f_{\Sigma_{2, \hat{k}}}(1-z) d z \\
&= O\left(m_{k}^{3}\right)+O\left(e^{-\frac{\sqrt{\lambda_{k}}}{2(\sqrt{\beta}+1)} \varepsilon^{2}}\right) \\
&+O\left(\frac{\varepsilon^{2}}{\lambda_{k}}\right)\left(\int_{0}^{1} g_{0}(z) f_{\Sigma_{2, \hat{k}}}(1-z) d z-O\left(m_{k}\right)\right) \\
&= O\left(m_{k}^{3}\right)+O\left(e^{-\frac{\sqrt{\lambda_{k}}}{2(\sqrt{\beta}+1)} \varepsilon^{2}}\right)+O\left(\frac{\varepsilon^{2}}{\lambda_{k}}\right) f_{S_{N, \lambda}}(1) .
\end{aligned}
$$

Substituting the last formula with

$$
\varepsilon=\frac{\log N}{\lambda_{k}^{1 / 4}}
$$

into (3.151), and taking into account (3.135) we obtain

$$
\mathbb{E}\left\{\left(X_{k}-m_{k}\right)^{2} \mid \sum_{i=1}^{N} X_{i}=1\right\}=O\left(\frac{(\log N)^{2}}{\lambda_{k}^{3 / 2}}\right) .
$$

This together with (3.147) yields as well

$$
\operatorname{Var}\left\{X_{k} \mid \sum_{i=1}^{N} X_{i}=1\right\}=O\left(\frac{(\log N)^{2}}{\lambda_{k}^{3 / 2}}\right),
$$

which by the identity (3.14) is equivalent to

$$
\operatorname{Var}\left(Y_{N+1-k}-Y_{N-k}\right)=O\left(\frac{(\log N)^{2}}{\lambda_{k}^{3 / 2}}\right)=O\left(\frac{(\log N)^{2}}{\left(k F_{0} N\right)^{3 / 2}}\right),
$$

for all $k \geq 2$, where the last equality is due to (3.139). Hence, (3.155) after transformation (3.4) yields (2.18).

3.7. Proof of Theorem 2.2 in the critical phase (c). Let $F=4 \beta N$. Then similar to the previous case, we consider for any $1 \leq k \leq N$

$$
\mathbb{E}\left\{X_{k} \mid \sum_{i=1}^{N} X_{k}=1\right\}=\int_{0}^{1} x \frac{f_{k, \lambda}(x) f_{S_{N, \hat{k}, \lambda}}(1-x)}{f_{S_{N, \lambda}}(1)} d x .
$$


Here, as in (3.141),

$$
f_{k, \lambda}(x)=\frac{e^{-\frac{\beta}{x}-\lambda_{k} x}}{I_{1}\left(\lambda_{k}, \beta\right)+e^{-\lambda_{k} / 2}},
$$

with $\lambda$ defined in Lemma 3.5 [(b) Critical force], so that

$$
\lambda_{1}=4 \beta N+\lambda \geq a N,
$$

and for all $k \geq 2$

$$
\lambda_{k}=4 \beta N k+\lambda>4 \beta N(k-1)>4 \beta N .
$$

Thus, by Proposition 3.3 we have here

$$
m_{k}=E X_{k, \lambda}=\sqrt{\frac{\beta}{\lambda_{k}}}(1+o(1))=\sqrt{\frac{1}{4 N(k-1)+\Theta(N)}}(1+o(1))
$$

where $o(1)$ is uniform in $k$ (since $\lambda_{k} \rightarrow \infty$ inform in $k$ ).

Using bound (3.142), we derive [as in (3.144)] for any positive $\varepsilon=o(1)<1$,

$$
\begin{aligned}
\int_{0}^{1} x & f_{k, \lambda}(x) f_{S_{N, \hat{k}, \lambda}}(1-x) d x \\
= & \int_{\left|x-m_{k}\right|>\varepsilon / \sqrt{\lambda_{k}}} x f_{k, \lambda}(x) f_{S_{N, \hat{k}, \lambda}}(1-x) d x \\
& +\int_{\left|x-m_{k}\right| \leq \varepsilon / \sqrt{\lambda_{k}}} x f_{k, \lambda}(x) f_{S_{N, \hat{k}, \lambda}}(1-x) d x \\
= & O\left(e^{-\frac{\sqrt{\lambda_{k}} \varepsilon^{2}}{2(\sqrt{\beta}+1)}}\right)+\left(m_{k}+O\left(\frac{\varepsilon}{\sqrt{\lambda_{k}}}\right)\right)\left(f_{S_{N, \lambda}}(1)-O\left(e^{-\frac{\sqrt{\lambda_{k}} \varepsilon^{2}}{2(\sqrt{\beta}+1)}}\right)\right) \\
= & \left(m_{k}+O\left(\frac{\varepsilon}{\sqrt{\lambda_{k}}}\right)\right) f_{S_{N, \lambda}}(1)+O\left(e^{-\frac{\sqrt{\lambda_{k}} \varepsilon^{2}}{2(\sqrt{\beta}+1)}}\right) .
\end{aligned}
$$

Choose

$$
\varepsilon=\frac{\log N}{\lambda_{k}^{1 / 4}}
$$

and substitute (3.160) with this value into (3.156) [taking into account (3.135)]. This gives us

$$
\mathbb{E}\left\{X_{k} \mid \sum_{i=1}^{N} X_{i}=1\right\}=m_{k}+O\left(\frac{\log N}{\lambda_{k}^{3 / 4}}\right)
$$


which by the identity (3.14) [and formula (3.159)] is equivalent to

$$
\begin{aligned}
\mathbb{E}\left(Y_{N+1-k}-Y_{N-k}\right) & =m_{k}+O\left(\frac{\log N}{\lambda_{k}^{3 / 4}}\right) \\
& =\sqrt{\frac{\beta}{(k-1) F+\Theta(N)}}+O\left(\frac{\log N}{(k N)^{3 / 4}}\right) .
\end{aligned}
$$

Hence, applying here transformation (3.4) we confirm (2.13).

Next, under the condition $F=4 \beta N$, consider

$$
\mathbb{E}\left\{\left(X_{k}-m_{k}\right)^{2} \mid \sum_{i=1}^{N} X_{k}=1\right\}=\int_{0}^{1}\left(x-m_{k}\right)^{2} \frac{f_{k, \lambda}(x) f_{S_{N, \hat{k}, \lambda}}(1-x)}{f_{S_{N, \lambda}}(1)} d x
$$

With exactly same argument as we derived (3.160), we get

$$
\begin{aligned}
\int_{0}^{1}\left(x-m_{k}\right)^{2} f_{k, \lambda}(x) f_{S_{N, \hat{k}, \lambda}}(1-x) d x \\
=O\left(e^{-\frac{\sqrt{\lambda_{k}} \varepsilon^{2}}{2(\sqrt{\beta}+1)}}\right)+O\left(\frac{\varepsilon^{2}}{\lambda_{k}}\right)\left(f_{S_{N, \lambda}}(1)-O\left(e^{-\frac{\sqrt{\lambda_{k}} \varepsilon^{2}}{2(\sqrt{\beta}+1)}}\right)\right) \\
=O\left(\frac{\varepsilon^{2}}{\lambda_{k}}\right) f_{S_{N, \lambda}}(1)+O\left(e^{-\frac{\sqrt{\lambda_{k}} \varepsilon^{2}}{2(\sqrt{\beta}+1)}}\right) .
\end{aligned}
$$

Setting here $\varepsilon=\frac{\log N}{\lambda_{k}^{1 / 4}}$ and using the result in (3.163), we obtain

$$
\mathbb{E}\left\{\left(X_{k}-m_{k}\right)^{2} \mid \sum_{i=1}^{N} X_{k}=1\right\}=O\left(\frac{(\log N)^{2}}{\lambda_{k}^{3 / 2}}\right),
$$

which together with (3.161) gives us as

$$
\operatorname{Var}\left\{X_{k} \mid \sum_{i=1}^{N} X_{k}=1\right\}=O\left(\frac{(\log N)^{2}}{\lambda_{k}^{3 / 2}}\right)=O\left(\frac{(\log N)^{2}}{(k N)^{3 / 2}}\right),
$$

where the last equality is due to (3.158). This by the identity (3.14) is equivalent to

$$
\operatorname{Var}\left(Y_{N+1-k}-Y_{N-k}\right)=O\left(\frac{(\log N)^{2}}{(k N)^{3 / 2}}\right),
$$

and thus (2.14) follows after transformation (3.4).

3.8. Proof of Theorem 2.2 in the supercritical phase (e). Finally, assume that $F \gg N$. Consider

$$
\mathbb{E}\left\{X_{1} \mid \sum_{i=1}^{N} X_{k}=1\right\}=\int_{0}^{1} x \frac{f_{1, \lambda}(x) f_{\Sigma_{2}}(1-x)}{f_{S_{N, \lambda}}(1)} d x
$$


where $\Sigma_{2}:=S_{N, \lambda}-X_{1, \lambda}$. In (3.165),

$$
f_{1, \lambda}(x)=\frac{e^{-\lambda_{1} x-\frac{\beta}{x}}}{\int_{0}^{1} e^{-\lambda_{1} x-\frac{\beta}{x}} d x}=\frac{e^{-(\lambda+F) x-\frac{\beta}{x}}}{\int_{0}^{1} e^{-(\lambda+F) x-\frac{\beta}{x}} d x},
$$

where by Lemma 3.5 [(c) Strong force] and Remark 3.6,

$$
0<-\lambda_{1}=-(\lambda+F)=\lambda_{0}+O\left(\frac{1}{\sqrt{F}}\right)=\Theta\left((F / N)^{1 / 4}\right) \rightarrow+\infty .
$$

Recall also that by (3.61)

$$
\mathbb{E} X_{1, \lambda}=1-\sqrt{\frac{4 \beta N}{F}}+O\left(\frac{1}{\sqrt{F}}\right)
$$

while

$$
\varepsilon:=\mathbb{E} \Sigma_{2}=\sqrt{\frac{4 \beta N}{F}}
$$

and by Corollary 3.11 part (III)

$$
\operatorname{Var}\left(\Sigma_{2}\right)=O\left(\frac{1}{F^{3 / 2}}\right)
$$

Let us rewrite the expectation in (3.165) as follows:

$$
\mathbb{E}\left\{X_{1} \mid \sum_{i=1}^{N} X_{k}=1\right\}=1-\frac{1}{f_{S_{N, \lambda}}(1)} \int_{0}^{1} x f_{\Sigma_{2}}(x) f_{1, \lambda}(1-x) d x .
$$

Then we obtain a simple bound

$$
\begin{aligned}
\int_{0}^{1} x & f_{\Sigma_{2}}(x) f_{1, \lambda}(1-x) d x \\
& =\int_{0}^{2 \varepsilon} x f_{\Sigma_{2}}(x) f_{1, \lambda}(1-x) d x+\int_{2 \varepsilon}^{1} x f_{\Sigma_{2}}(x) f_{1, \lambda}(1-x) d x \\
& \leq 2 \varepsilon f_{S_{N, \lambda}}(1)+\max _{0<y<1-2 \varepsilon} f_{1, \lambda}(y) \mathbb{P}\left\{\Sigma_{2}>2 \varepsilon\right\},
\end{aligned}
$$

where by (3.166) and (3.167)

$$
\max _{0<y<1-2 \varepsilon} f_{1, \lambda}(y)=O\left(\left|\lambda_{1}\right| e^{-\varepsilon\left|\lambda_{1}\right|}\right)=O(1 / \varepsilon),
$$

and by Chebyshev's inequality and (3.169),

$$
\mathbb{P}\left\{\Sigma_{2}>2 \varepsilon\right\} \leq \frac{\operatorname{Var}\left(\Sigma_{2}\right)}{\varepsilon^{2}}=O\left(\frac{1}{F^{3 / 2} \varepsilon^{2}}\right) .
$$


Substituting the last bounds into (3.171), we derive taking into account (3.135)

$$
\int_{0}^{1} x f_{\Sigma_{2}}(x) f_{1, \lambda}(1-x) d x=O(\varepsilon)+O\left(\frac{1}{F^{3 / 2} \varepsilon^{3}}\right)=O\left(\sqrt{\frac{N}{F}}\right)+O\left(N^{-3 / 2}\right) .
$$

Making use of the last formula and (3.135), we obtain from (3.170)

$$
\mathbb{E}\left\{X_{1} \mid \sum_{i=1}^{N} X_{k}=1\right\}=1-O\left(\sqrt{\frac{N}{F}}+N^{-3 / 2}\right) .
$$

This by the identity (3.14) is equivalent to

$$
\mathbb{E}\left(Y_{N}-Y_{N-1}\right)=1-O\left(\sqrt{\frac{N}{F}}+N^{-3 / 2}\right) .
$$

Following exactly same approach, we also derive

$$
\begin{aligned}
\mathbb{E}\left\{\left(X_{1}-1\right)^{2} \mid \sum_{i=1}^{N} X_{k}=1\right\} \\
=\frac{1}{f_{S_{N, \lambda}}(1)} \int_{0}^{1} x^{2} f_{\Sigma_{2}}(x) f_{1, \lambda}(1-x) d x \\
=O\left(\varepsilon^{2}\right)+O\left(\frac{1}{F^{3 / 2} \varepsilon^{3}}\right)=O\left(\frac{N}{F}\right)+O\left(N^{-3 / 2}\right) .
\end{aligned}
$$

This together with the identity (3.14) and (3.173) gives us

$$
\operatorname{Var}\left(Y_{N-1}\right)=O\left(\frac{N}{F}+N^{-3 / 2}\right) \text {. }
$$

Bounds (3.174) and (3.176) confirm, correspondingly both statements of Theorem 2.2 in the phase (e).

The theorem is proved.

Acknowledgements. The author thanks V. Malyshev for introducing this problem, and Y. Ameur for the helpful and inspiring discussions on the general framework of this model. The author also gratefully acknowledges discussions with D. Mason, G. Napolitano and B. Söderberg.

Finally, the author thanks the reviewers for many constructive comments.

\section{REFERENCES}

[1] Aizenman, M. and Martin, P. A. (1980). Structure of Gibbs states of one dimensional Coulomb systems. Comm. Math. Phys. 78 99-116.

[2] Ameur, Y., Hedenmalm, H. and Makarov, N. (2015). Random normal matrices and Ward identities. Ann. Probab. 43 1157-1201.

[3] Ameur, Y., KANG, N.-G. and MAKAROV, N. Rescaling Ward identities in the random normal matrix model. arXiv:1410.4132. 
[4] Bauerschmidt, R., Bourgade, P., Nikula, M. and Yau, H.-T. Local density for twodimensional one-component plasma. arXiv: 1510.02074 .

[5] Bauerschmidt, R., Bourgade, P., Nikula, M. and Yau, H.-T. The two-dimensional Coulomb plasma: Quasi-free approximation and central limit theorem. arXiv:1609.08582.

[6] Cunden, F. D., FACCHI, P. and Vivo, P. (2016). A shortcut through the Coulomb gas method for spectral linear statistics on random matrices. J. Phys. A: Math. Theor. 4935202.

[7] Diaconis, P. and Freedman, D. A. (1988). Conditional limit theorems for exponential families and finite versions of de Finetti's theorem. J. Theoret. Probab. 1 381-410. MR0958245

[8] EdWARdS, S. F. and LENARD, A. (1962). Exact statistical mechanics of a one-dimensional system with Coulomb forces. II. The method of functional integration. J. Math. Phys. 3 778-792. MR0147214

[9] FedoryuK, M. V. (1987). Asimptotika: Integraly i Ryady. Nauka, Moscow. (Russian) [Asymptotics: Integrals and Series].

[10] Feller, W. (1971). An Introduction to Probability Theory and Its Applications, Vol. 2. Wiley.

[11] JohAnsson, K. (1991). Separation of phases at low temperatures in a one-dimensional continuous gas. Comm. Math. Phys. 141 259-278.

[12] Johansson, K. (1995). On separation of phases in one-dimensional gases. Comm. Math. Phys. 169 521-561.

[13] Leblé, T., Serfaty, S. and Zeitouni, O. (2017). Large deviations for the two-dimensional two-component plasma. Comm. Math. Phys. 350 301-360.

[14] Lenard, A. (1961). Exact statistical mechanics of a one-dimensional system with Coulomb forces. J. Math. Phys. 2682.

[15] LenARD, A. (1963). Exact statistical mechanics of a one-dimensional system with Coulomb forces. III. Statistics of the electric field. J. Math. Phys. 4533.

[16] Malyshev, V. A. (2015). Phase transitions in the one-dimensional Coulomb medium. Probl. Inf. Transm. 51 31-36.

[17] Malyshev, V. A. and Zamyatin, A. A. (2015). One-dimensional Coulomb multiparticle systems. Adv. Math. Phys.

[18] Petrov, V. V. (1975). Sums of Independent Random Variables. Springer.

[19] Serfaty, S. (2015). Coulomb Gases and Ginzburg-Landau Vortices. European Mathematical Society. 